University of Zurich

Department of Economics

Working Paper Series

ISSN 1664-7041 (print)

ISSN 1664-705X (online)

Working Paper No. 179

\title{
The Two Faces of Independence: Betweenness and Homotheticity
}

Daniel R. Burghart, Thomas Epper and Ernst Fehr

November 2014 


\title{
The Two Faces of Independence: Betweenness and Homotheticity
}

\author{
Daniel R. Burghart* Thomas Epper ${ }^{\dagger} \quad$ Ernst Fehr ${ }^{\ddagger}$
}

October 22, 2014

\begin{abstract}
Many studies document failures of expected utility's key assumption, the independence axiom. Here, we show that independence can be decomposed into two distinct axioms betweenness and homotheticity - and that these two axioms are necessary and sufficient for independence. Thus, independence can fail because homotheticity, betweenness, or both are violated. Most research has focused on models that assume subjects will violate both axioms or models that assume subjects will satisfy betweenness but violate homotheticity. Our decomposition of independence into betweenness and homotheticity allows us to show, however, that a significant share of subjects obey homotheticity but violate betweenness.

Using data from a revealed preference experiment, and without making any parametric assumptions, we show that $1 / 3$ of participants belong in the neglected class of preferences that violate independence but satisfy homotheticity, indicating that betweenness is violated. Another $1 / 3$ of participants satisfy independence. The remaining $1 / 3$ fail both independence and homotheticity and may also fail betweenness. Our results provide useful constraints on future modeling attempts by highlighting, in a non-parametric way, an empirically relevant class of preferences.
\end{abstract}

JEL Codes: C91, D11, D12, D83

Keywords: Revealed preferences, risk preferences, expected utility, independence axiom, betweenness, homotheticity, consumer choice, aggregation.

\footnotetext{
*Corresponding author. University of Zurich, Department of Economics, Bluemlisalpstrasse 10, 8006 Zurich, Switzerland. dan.burghart@econ.uzh.ch

${ }^{+}$University of Zurich, Department of Economics, Bluemlisalpstrasse 10, 8006 Zurich, Switzerland. thomas.epper@econ.uzh.ch

‡University of Zurich, Department of Economics, Bluemlisalpstrasse 10, 8006 Zurich, Switzerland. ernst.fehr@econ.uzh.ch All authors gratefully acknowledge valuable input from Charles Efferson, Helga Fehr-Duda, Jan Heufer, Holger Herz, John Hey, Michael Powell, Collin Raymond, Uzi Segal, Mike Visser, Roberto Weber, Tony Williams, and participants in the micro workshops at UZH. This paper has been dramatically improved because of extensive comments provided by Peter Wakker. This work was supported by a grant from the European Research Council (\#295642).
} 
The normative benchmark for decision-making under risk is expected utility. Expected utility's key assumption is the independence axiom. Graphically, the independence axiom gives rise to indifference curves that are linear and parallel in probability space. A weakening of independence is the betweenness axiom. Betweenness relaxes the parallelism restriction of independence but retains linearity (Dekel, 1986; Chew, 1989). Betweenness's relaxation of the parallelism restriction embedded in the independence axiom hints that there is another alternative to independence, an alternative that retains parallelism but relaxes linearity.

In this paper we introduce a parallelism condition that does not require linearity: homotheticity. Homotheticity is a widely invoked parallelism condition in traditional microeconomic analysis because of its role in aggregation theorems - the existence of a representative agent is assured only when every consumer in an economy has homothetic preferences (Gorman, 1953; Chipman, 1974; Polemarchakis, 1983). We adapt traditional homotheticity to the study of riskychoices by having the worst outcome under consideration serve as the homothetic center (origin) from which indifference curves expand and contract. ${ }^{1}$

We establish in Proposition 1 that invoking both homotheticity and betweenness is equivalent to invoking independence - betweenness and homotheticity are necessary and sufficient conditions for the independence axiom. The proposition decomposes independence into two distinct principles: One principle restricts indifference curves to be linear in the probability space (betweenness), the other principle restricts indifference curves to be parallel (homotheticity).

Decomposing the independence axiom into distinct principles allows for a better understanding of how independence can fail. Knowing whether homotheticity fails, betweenness fails, or both fail, is relevant for selecting theories of choice under risk. Theories in the betweenness class predict that homotheticity will fail (with the exception of the special case of expected utility). Other theories predict that both betweenness and homotheticity will fail. For example, typical parameterizations of rank-dependent theories, such as rank-dependent utility (Quiggin, 1982) and cumulative prospect theory (Tversky and Kahneman, 1992), generate indifference curves that are both nonparallel (i.e. a violation of homotheticity) and nonlinear (i.e. a violation of between-

\footnotetext{
${ }^{1}$ Having the worst outcome serve as the origin and homothetic center has strong appeal. In traditional microeconomic analysis the worst commodity bundle available is the origin (i.e. $\mathbf{0} \in \mathbb{R}_{+}^{L}$ ) and is also the homothetic center.
} 
ness). This indicates that typical parameterizations of rank-dependent utility and cumulative prospect theory entangle violations of betweenness and homotheticity. This entanglement could be called into question if some people violate only betweenness, or only homotheticity. Such patterns of behavior would also suggest serious consideration for a class of risky-choice models that have not gained much attention in the past. In our search of the literature, we could find only three papers that specifically explore models satisfying homotheticity, but not betweenness. And these papers have received little attention when compared to rank-dependent utility, cumulative prospect theory, and models explicitly in the betweenness class. ${ }^{2}$ Our results indicate, however, that more theoretical attention should be paid to preferences in the homothetic, non-betweenness class.

Assessing whether people violate betweenness, homotheticity, or both, is an empirical concern. And our decomposition of independence into betweenness and homotheticity provides an indirect empirical pathway for testing whether people violate only betweenness. Specifically, if preferences violate independence but satisfy homotheticity it is because betweenness is violated. This indirect method of assessing betweenness can only be accomplished when direct assessments of homotheticity and independence are possible. To insure this possibility we conducted a lab experiment. Our experimental design systematically varied a constant tradeoff between probabilities for three-outcome lotteries. We interpret this constant tradeoff as a price along a linear (Walrasian) budget in probability space. And it follows that choices from these budgets can be interpreted as traditional Marshallian demands. Interpreting lottery choices as traditional demands means that we can analyze choices using non-parametric revealed preference tests of necessity and sufficiency. This analysis indicates that about $1 / 3$ of the people in our sample made choices consistent with expected utility's key assumption - their choices conformed to the independence axiom. For the $2 / 3$ 's of our sample that violated independence, about $1 / 2$ (i.e.

\footnotetext{
${ }^{2}$ As we discuss more thoroughly in Section 1.4, Diecidue, Schmidt, and Zank (2009), Safra and Segal (1998), and Grant and Kajii (1998) all examine models that invoke some form of homotheticity, but not betweenness. To the extent that citation counts are indicative of the attention that this class of models have received, Google Scholar (as of September 1, 2014) indicates that these three papers have jointly accumulated only 118 citations (33, 71, and 14, respectively). In contrast, disappointment aversion theory (Gul, 1991), which is in the class of models that satisfy betweenness but not homotheticity, has garnered more than 800 citations. In the class of non-betweenness and nonhomothetic models, two popular examples are rank-dependent utility (Quiggin, 1982) and cumulative prospect theory (Tversky and Kahneman, 1992). These two papers have received more than 2,100 and 6,900 citations, respectively.
} 
1/3 of the full sample) made choices consistent with homotheticity. As these peoples' choices violated independence, but satisfied homotheticity, betweenness had to be violated. The rest of the people in our sample made choices inconsistent with both independence and homotheticity.

The remainder of our paper is divided into four sections. The next section introduces key terms and explores how our work is connected to the existing literature. Section 2 goes over our experimental design and sampling methodologies. Section 3 lays out each of our tests and our empirical findings. The final section discusses some implications of our results and concludes. 


\section{Preliminaries and Background}

We focus on lotteries over a set of finite, fixed, monetary outcomes, $z_{1}>z_{2}>\cdots>z_{J}$. Denote a lottery as a $J$-tuple $\left(p_{1}, p_{2}, \ldots, p_{J}\right)$, where each element assigns probability mass to its respective outcome. The states to which probabilities are assigned are mutually exclusive and exhaustive. Let $\Delta$ be the set of all such lotteries (probability distributions). Formally,

$$
\Delta \equiv\left\{\left(p_{1}, p_{2}, \ldots, p_{J}\right) \in \mathbb{R}_{+}^{J} \mid \sum_{j=1}^{J} p_{j}=1\right\} .
$$

We will discuss specific examples, and provide illustrations for lotteries over three, nonnegative outcomes. The set of these lotteries can be graphically represented in the probability simplex (see Figure 1, Marschak, 1950, and Machina, 1982). The vertical leg represents the probability $p_{1}$ that the best outcome $z_{1}$ materializes; the horizontal leg represents the probability $1-p_{3}=p_{1}+p_{2}$ that the worst outcome $z_{3}$ does not materialize. At the upper-right, lower-right, and lower-left vertices of the triangle, the probability assigned to each of the outcomes $z_{1}, z_{2}$, and $z_{3}$ is, respectively, equal to unity. Lotteries along the edges of the triangle assign probability mass to only two of the three outcomes while lotteries on the interior of the triangle (the gray area in Figure 1) assign strictly positive probability mass to all three outcomes.

In what follows, preferences over lotteries $(\succsim)$ are assumed to be imbued with the following properties:

- Completeness: For every $A, B \in \Delta, A \succsim B, B \succsim A$, or both.

- Transitivity: For $A, B, C \in \Delta$, if $A \succsim B$, and $B \succsim C$, then $A \succsim C$.

- Continuity: For every pair of convergent sequences of lotteries, $A^{n} \rightarrow A$, and $B^{n} \rightarrow B$, if $A^{n} \succsim B^{n}$ for every $n$, then $A \succsim B$.

- Monotonicity: Denoting $p_{j}^{A}$ and $p_{j}^{B}$ as the $j^{\text {th }}$ entries of $A, B \in \Delta$, if, for every $j, \sum_{j} p_{j}^{A} \geq \sum_{j} p_{j}^{B}$, with strict inequality for at least one $j$, then $A \succ B$. 
Figure 1: The Probability Triangle

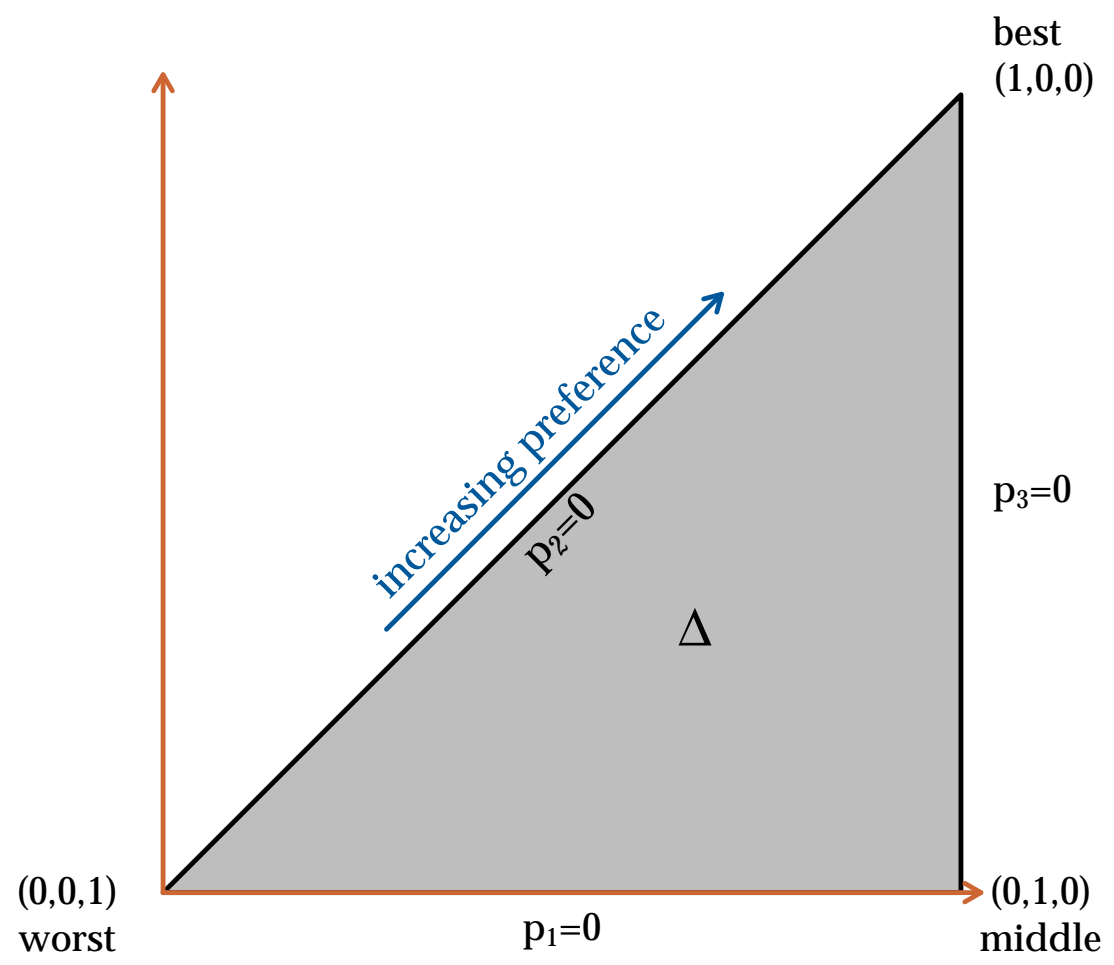




\subsection{The independence axiom}

The expected utility theorem establishes that preferences over lotteries $(\succsim)$ admit an expected utility representation $\left(\sum_{j} p_{j} u_{j}\right)$ if and only if preferences adhere to the independence axiom:

Axiom 1. The preference relation $\succsim$ satisfies independence if, for all lotteries $A, B, C \in \Delta$ and any $\alpha \in[0,1]$ we have

$$
A \sim B \Leftrightarrow \alpha A+(1-\alpha) C \sim \alpha B+(1-\alpha) C
$$

In words, independence requires that a preference ordering between two lotteries is unchanged when mixing those lotteries with a third. One can interpret independence as requiring preferences for probabilities to have no complementarities. Indeed, Marschak (1951) and Samuelson (1952) defended independence on normative grounds because the lack of complementarities is embedded in the objects of risky choice - only one of the outcomes from a lottery will materialize and be consumed (see also the discussion in Sugden (2004), Section 2.2). Thus, preferences for probabilities of these mutually exclusive outcomes should respect this fact. But others made ob-

jections to independence, the most prominent of which is due to Maurice Allais $(1953,1978)$ (see also Manne (1952) and Wold, Shackle, and Savage (1952). Fishburn and Wakker (1995) provide a comprehensive historical review of the independence assumption).

\subsection{The Allais paradox}

Various experimental studies have examined Allais-type common-ratio violations (see Fehr-Duda and Epper (2012) for a recent review). In Kahneman and Tversky (1979)'s Problems 3 and 4, for example, experimental participants were offered a choice between a lottery with an $80 \%$ chance of receiving $\$ 4,000$ and a $20 \%$ chance of receiving $\$ 0$ [ $A=(0.80,0.00,0.20)]$ or, a certain $\$ 3,000$ $[B=(0,1,0)]$. When surveyed, the majority of people indicated a strong preference for the certain \$3,000 (i.e. $B \succ A$ ). The participants were also presented with another pair of lotteries: a $20 \%$ chance of receiving $\$ 4,000$ and an $80 \%$ chance of receiving $\$ 0\left[A^{\prime}=(0.20,0.00,0.80)\right]$. Or, a $25 \%$ chance of receiving $\$ 3,000$ and a $75 \%$ chance of receiving $\$ 0\left[B^{\prime}=(0.00,0.25,0.75)\right]$. The lotteries $A^{\prime}$ and $B^{\prime}$ were constructed as mixtures with the worst outcome. Specifically, $A^{\prime}=\alpha A+(1-\alpha) C$ 
and $B^{\prime}=\alpha B+(1-\alpha) C$ with $\alpha=0.25$ and $C=(0,0,1)$. Given that $B \succ A$, behavior consistent with independence requires that $B^{\prime} \succ A^{\prime}$. When surveyed, however, most people indicated the opposite preference (i.e. $A^{\prime} \succ B^{\prime}$ ), a clear violation of independence.

Allais-type violations of independence can be illustrated using the probability triangle. In Panel (a) of Figure 2, the endpoints of the red line-segment depict the lotteries available in Kahneman and Tversky (1979) Problem 3: $\{A, B\}$. If preferences satisfy independence then indifference curves must be parallel and straight-lines in the simplex. Thus, if $B \succ A$, independence-satisfying indifference curves must have a slope that exceeds that of the red line-segment. Kahneman and Tversky (1979) Problem $4,\left\{A^{\prime}, B^{\prime}\right\}$, is depicted as the endpoints of the blue line segment. Note that this blue line-segment has the same slope as the red line-segment. Because indifference curves that satisfy independence must be parallel (and linear), the slope of an independencesatisfying indifference curve that rationalizes the original choice $(B \succ A)$ must also exceed the slope of the blue line-segment. Therefore, if preferences satisfy independence, we should observe $B^{\prime} \succ A^{\prime}$, instead of $A^{\prime} \succ B^{\prime}$.

\subsection{The betweenness axiom}

The betweenness axiom can accommodate Allais-type behavior by relaxing independence:

Axiom 2. The preference relation $\succsim$ satisfies betweenness if, for all lotteries $A, B \in \Delta$ and any $\alpha \in[0,1]$ we have

$$
A \sim B \Leftrightarrow A \sim \alpha A+(1-\alpha) B \sim B
$$

Betweenness requires that a probability mixture of two lotteries must lie between them in preference. Such preferences belong to the linear equivalence class. Several models have been proposed that build on the betweenness axiom. Gul (1991)'s disappointment aversion and implicit weighted utility (Chew and Epstein, 1989; Dekel, 1986) are prominent examples.

Graphically, the betweenness axiom requires indifference curves to be straight-lines but removes the parallelism requirement of independence. The gray lines in Panel (b) of Figure 2 depict an indifference map that satisfies betweenness but not independence; the indifference curves fan 
Figure 2: Allais common ratio choice problems

(a) Independence cannot rationalize Allais-type behavior $\left(B \succ A\right.$ and $\left.A^{\prime} \succ B^{\prime}\right)$

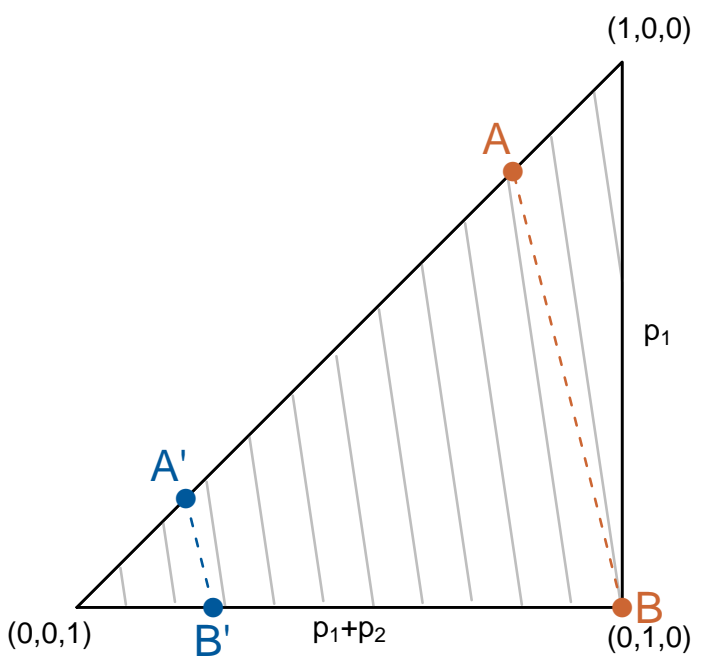

(c) Indifference curves that satisfy homotheticity, but not betweenness, cannot rationalize Allais-type behavior

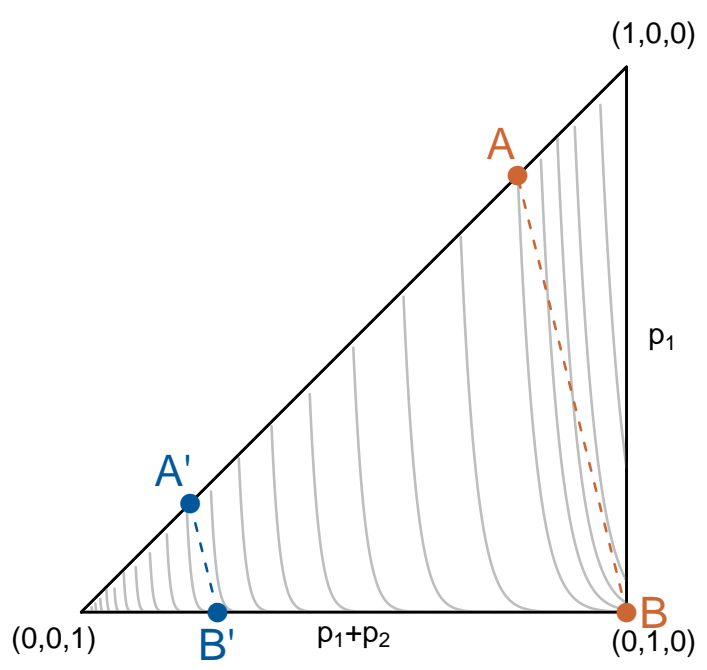

(b) Betweenness can rationalize Allais-type behavior

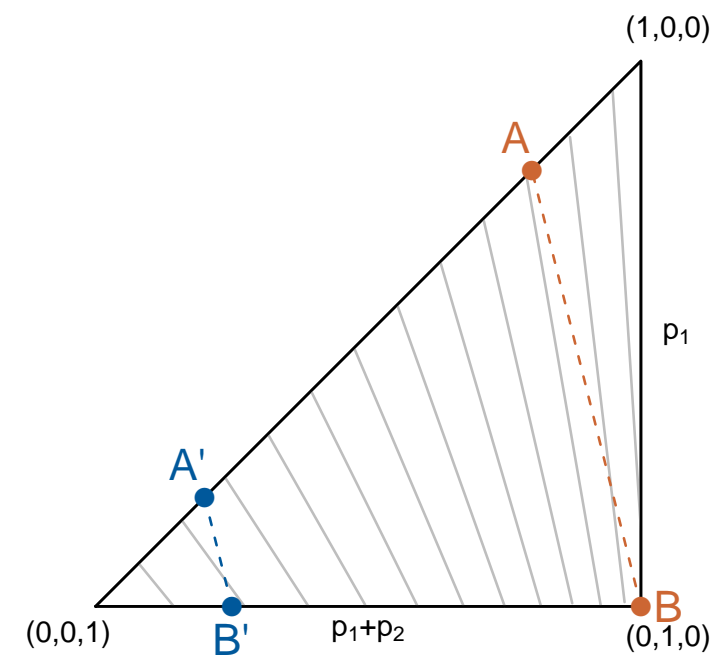

(d) A generalized Allais-type choice problem that includes a mixture alternative

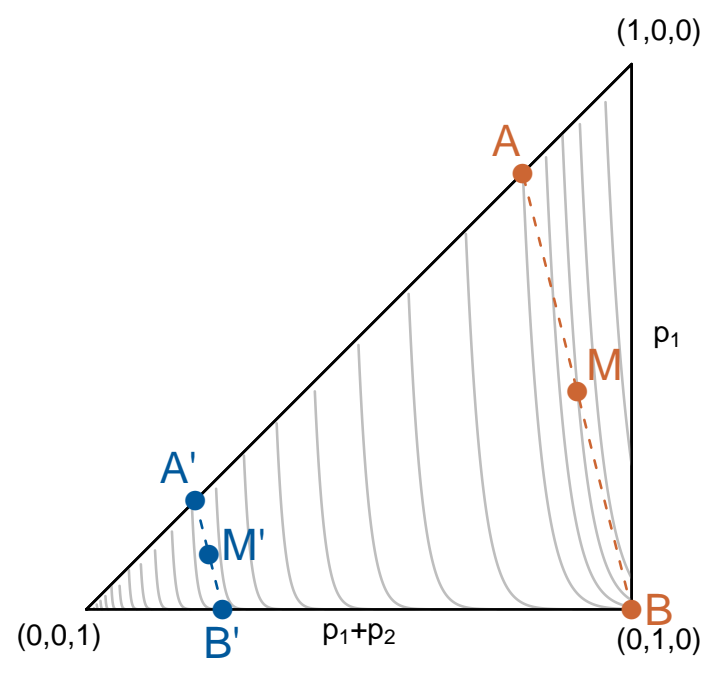


out across the simplex (see Machina (1982) hypothesis II). Note that for this indifference map Allais-type violations of independence can be accommodated.

\subsection{Homotheticity}

Straight-line, but not parallel, indifference curves consistent with betweenness can rationalize Allais-type behavior. This suggests that Allais-type violations of independence are, in fact, violations of the parallelism assumption embedded in the independence axiom. We introduce here a parallelism assumption - homotheticity - that does not require indifference curves to be linear. Our homotheticity condition differs from traditional homotheticity in that it treats the worst outcome under consideration as the origin from which indifference curves expand and contract:

Axiom 3. The preference relation $\succsim$ satisfies homotheticity if, for all lotteries $A, B \in \Delta$ with $A \sim B$, the worst outcome $W=(0,0, \ldots, 1)=z_{J}$ in $\Delta$ (i.e. $\forall A \in \Delta \backslash W, A \succ W$ ) and any $\alpha \in[0,1]$, we have

$$
A \sim B \Leftrightarrow \alpha A+(1-\alpha) W \sim \alpha B+(1-\alpha) W
$$

Homotheticity restricts preferences such that the ordering between two lotteries is retained when mixing both with the worst possible outcome. Indeed, this is exactly the assumption tested by the common ratio examples: The second choice pair, $A^{\prime}$ and $B^{\prime}$, is obtained by mixing the original lotteries $A$ and $B$ with the worst possible lottery $C=(0,0,1)$. Homotheticity is less restrictive than independence. For example, the indifference map depicted by the gray curves in Panel (c) of Figure 2 conform to homotheticity - they are parallel expansions of each other from the origin. These curves, however, are non-linear and therefore they are inconsistent with the linearity principle of independence and betweenness.

In contrast to independence and betweenness, homotheticity is flexible enough to permit a preference for, or aversion to, probability mixtures. To discuss this issue more precisely we introduce the following terms by taking $A, B \in \Delta$ with $A \sim B$ :

- Quasi-concavity: $\alpha A+(1-\alpha) B \succsim A$, for every $\alpha \in[0,1]$

- Quasi-convexity: $A \succsim \alpha A+(1-\alpha) B$, for every $\alpha \in[0,1]$ 
We will refer to strict quasi-concavity/convexity by interchanging the weak preference relation $(\succsim)$ with the strict preference relation $(\succ)$. Quasi-concavity guarantees that upper contour sets, $\{C \in \Delta \mid C \succsim A\}$, are convex sets. In this way, preferences that adhere to quasi-concavity will exhibit a preference for probability mixtures. Quasi-convexity is the reverse: Lower contour sets, $\{C \in \Delta \mid A \succsim C\}$, are convex sets, which accommodates an aversion to probability mixtures. If the two binary choice-situations in Allais' experiment are generalized to include a third alternative, an alternative that is a probability mixture of the two end-points of the line segments, people who exhibit a preference for mixtures will prefer these lotteries. Panel (d) of Figure 2 illustrates homothetic, quasi-concave preferences graphically - the indifference map depicted is consistent with individuals selecting the mixture alternatives $\left(M\right.$ and $\left.M^{\prime}\right)$ in these generalized Allais-type choice-situations.

Previous studies have identified conditions that are quite similar to homotheticity. Diecidue, Schmidt, and Zank (2009) identify a condition they call common ratio invariance for decumulative probability distributions that has the same principle as homotheticity: "Common ratio invariance for decumulative distributions says that shifting proportionally probability mass from good consequences to the worst consequence (or doing the opposite) leaves preferences unaffected, which precisely rules out the common ratio effect [Allais-type violations of independence]" (Diecidue, Schmidt, and Zank (2009), p.1107). Their study establishes how common ratio invariance is the critical assumption for a rank-dependent utility model with a power weighting function (i.e. $w(p)=p^{\gamma}$ with $\gamma \neq 0$ ), a model that is well-known to rule out Allais-type violations of independence, but permits a strict preference for $(\gamma<0)$ or aversion to $(\gamma>0)$ probability mixtures. Diecidue, Schmidt, and Zank (2009) did not, however, establish a connection between common ratio invariance and homotheticity conditions commonly invoked in traditional microeconomic analysis, nor did they identify the role that homotheticity can play in relation to the independence axiom, when it is coupled with the betweenness axiom, a novel result we document in this paper.

The paper by Safra and Segal (1998) contains a similar condition to homotheticity that is called zero independence. Zero independence requires preference orderings to be maintained when lotteries are mixed with a degenerate, zero outcome, the worst outcome in Safra and Segal (1998)'s 
configuration. Safra and Segal (1998) demonstrates that "Together with constant risk aversion, this axiom [zero independence] implies Yaari's representation with a probability transformation function of the [power] form $g(p)=1-(1-p)^{t \prime \prime}$ (Safra and Segal (1998), p.21; brackets added). Safra and Segal (1998)'s model can accommodate a strict preference for probability mixtures while ruling out Allais-type violations of independence. This behavioral pattern can also be captured by some well-known models that involve direct transformation of probabilities, such as the theories introduced by Edwards (1962), Handa (1977), and original prospect theory (Kahneman and Tversky, 1979). While these models can satisfy homotheticity, this comes at the cost of violations of monotonicity or transitivity, a theoretical tradeoff few economists are willing to accept.

In a rank-dependent utility setting, Grant and Kajii (1998) invoke a condition similar to homotheticity that rules out common-ratio violations when lotteries are mixed with the worst outcome. They coin their condition the axiom of downward scale invariance. The focus of Grant and Kajii (1998) is, however, their axiom of upward scale invariance, a condition that rules out commonratio violations when lotteries are mixed with the best outcome. In this paper, we follow the standard setup for common-ratio choice pairs in which lotteries are mixed only with an inferior outcome (see, for example, Starmer, 2000, and Fehr-Duda and Epper, 2012)

For the present study, homotheticity (or common ratio invariance, or zero independence, or downward scale invariance) is particularly valuable as an empirically testable condition because it is a component of an indirect assessment of the betweenness axiom. As we document in section 1.6, if preferences satisfy homotheticity, but fail independence, it is because betweenness is violated. Thus, establishing that betweenness is violated requires direct assessments of both homotheticity and independence. A direct assessment of homotheticity is straightforward due to the linkage between it and traditional homotheticity assumptions - Varian's homothetic axiom of revealed preference provides a direct assessment of necessity and sufficiency for homotheticity.

We speculate that linking homotheticity to traditional homotheticity axioms, axioms that are critical for aggregation of preferences over commodity bundles, will prove to be theoretically valuable. Indeed, the paper by Rubinstein, Safra, and Thomson (1992) is suggestive that such a linkage contains value - Rubinstein, Safra, and Thomson (1992) invoke a related condition, 
homogeneity, on utility functions defined over payoffs in a game-theoretic setting. Homogeneity is then used for aggregating choices over mixed-strategies, the game-theoretic analogue of lotteries.

\subsection{The independence axiom: an entanglement of homotheticity and betweenness}

The independence axiom produces indifference curves that are straight-lines and parallel. Intuition suggests that combining the linearity of betweenness and the parallelism of homotheticity will produce an equivalency with independence. We formalize this intuition with a proposition:

Proposition 1. Assume that preferences conform to completeness, transitivity, continuity, and monotonicity. The preference relation $\succsim$ satisfies independence if and only if it satisfies betweenness and homotheticity.

Proof. It is widely known that independence implies betweenness. And it is easy to see that independence also implies homotheticity. To show that betweenness and homotheticity imply independence first assume that $V(\cdot)$ is a utility function representing preferences $(\succsim)$ that satisfy betweenness and homotheticity (in addition to completeness, transitivity, continuity, and monotonicity, of which the first three conditions guarantee the existence of a continuous utility function representing $\succsim)$. Take $A, B \in \Delta$ such that $A \sim B$. The betweenness axiom guarantees a constant marginal rate of substitution along a single indifference curve, a property that can be interpreted as guaranteeing an expected utility representation $\left(V(\cdot)=\sum p_{j} u_{j}\right)$ along that single indifference curve (Machina, 1982). We refer to this single indifference curve representation as a "local expected utility representation at $A / B$. ." Formally, betweenness implies:3

$$
\frac{\frac{\partial V(A)}{\partial p_{j}}}{\frac{\partial V(A)}{\partial p_{k}}}=\frac{\frac{\partial V(M)}{\partial p_{j}}}{\frac{\partial V(M)}{\partial p_{k}}}=\frac{\frac{\partial V(B)}{\partial p_{j}}}{\frac{\partial V(B)}{\partial p_{k}}}=\frac{u_{j}}{u_{k}}=c_{j k}
$$

where $M$ is a mixture of $A$ and $B$ (i.e. $M=\alpha A+(1-\alpha) B, \alpha \in[0,1]), j$ and $k$ are used to index all possible outcomes, and $u_{j}$ and $u_{k}$ are the utility numbers for the local expected utility representation at $A / B$.

\footnotetext{
3If preferences are complete, transitive, continuous, and also satisfy betweenness, it can be shown that any utility function representing these preferences will be continuously differentiable along the open interval $(A, B)$ when $A \sim B$. In this proof we assume that the derivatives at the endpoints of the interval $[A, B]$ are taken only in the direction of the interior of the interval, thus guaranteeing the constancy of the partial derivative along the closed interval $[A, B]$.
} 
Define $A^{\prime}$ and $B^{\prime}$ as homothetic mixtures with $A$ and $B$, respectively: $A^{\prime}=\alpha A+(1-\alpha) W$ and $B^{\prime}=\alpha B+(1-\alpha) W$, where $W$ is the worst outcome and $\alpha \in[0,1]$. Note that by homotheticity $A^{\prime} \sim B^{\prime}$. It is well known that for utility functions representing homothetic preferences that the marginal rate of substitution (MRS) is constant between homothetic mixtures. Thus:

$$
\frac{\frac{\partial V(A)}{\partial p_{j}}}{\frac{\partial V(A)}{\partial p_{k}}}=\frac{\frac{\partial V\left(A^{\prime}\right)}{\partial p_{j}}}{\frac{\partial V\left(A^{\prime}\right)}{\partial p_{k}}}, \quad \text { and } \quad \frac{\frac{\partial V(B)}{\partial p_{j}}}{\frac{\partial V(B)}{\partial p_{k}}}=\frac{\frac{\partial V\left(B^{\prime}\right)}{\partial p_{j}}}{\frac{\partial V\left(B^{\prime}\right)}{\partial p_{k}}}
$$

And once again we can rely on betweenness to guarantee constancy of the MRS at any mixture $\left(M^{\prime}\right)$ of $A^{\prime}$ and $B^{\prime}$ :

$$
\frac{\frac{\partial V\left(A^{\prime}\right)}{\partial p_{j}}}{\frac{\partial V\left(A^{\prime}\right)}{\partial p_{k}}}=\frac{\frac{\partial V\left(M^{\prime}\right)}{\partial p_{j}}}{\frac{\partial V\left(M^{\prime}\right)}{\partial p_{k}}}=\frac{\frac{\partial V\left(B^{\prime}\right)}{\partial p_{j}}}{\frac{\partial V\left(B^{\prime}\right)}{\partial p_{k}}}
$$

Collecting all of these equalities gives:

$$
\frac{\frac{\partial V(A)}{\partial p_{j}}}{\frac{\partial V(A)}{\partial p_{k}}}=\frac{\frac{\partial V(M)}{\partial p_{j}}}{\frac{\partial V(M)}{\partial p_{k}}}=\frac{\frac{\partial V(B)}{\partial p_{j}}}{\frac{\partial V(B)}{\partial p_{k}}}=\frac{\frac{\partial V\left(A^{\prime}\right)}{\partial p_{j}}}{\frac{\partial V\left(A^{\prime}\right)}{\partial p_{k}}}=\frac{\frac{\partial V\left(M^{\prime}\right)}{\partial p_{j}}}{\frac{\partial V\left(M^{\prime}\right)}{\partial p_{k}}}=\frac{\frac{\partial V\left(B^{\prime}\right)}{\partial p_{j}}}{\frac{\partial V\left(B^{\prime}\right)}{\partial p_{k}}}=\frac{u_{j}}{u_{k}}=c_{j k}
$$

Thus, the matrix of the marginal rates of substitution for $V(\cdot)$ is everywhere constant. Therefore the local expected utility representation at $A / B$ can be the same as the local expected utility representation at $A^{\prime} / B^{\prime}$. This shows that $V(\cdot)$ can be an expected utility for all of $\Delta$ and therefore, by the expected utility theorem, preferences $(\succsim)$ must satisfy independence. This demonstrates that betweenness and homotheticity imply independence and completes the proof.

Figure 3 illustrates the intuition behind this proof in the simplex. The thick line-segment represents a betweenness-satisfying indifference curve with $A \sim B$. Homotheticity requires that the mixtures $A^{\prime}$ and $B^{\prime}$ must also be indifferent. The thin line-segment represents an indifference curve with $A^{\prime} \sim B^{\prime}$ which, by betweenness, must also be linear. This illustrates how Proposition 1 decomposes independence into distinct axiomatic principles. One principle, betweenness, restricts indifference curves to be linear, but not parallel. The other principle, homotheticity, restricts indifference curves to be parallel, but not linear. When these two principles are combined, they give rise to an equivalence with expected utility's key assumption, independence. 
Figure 3: Betweenness and homotheticity are equivalent to independence

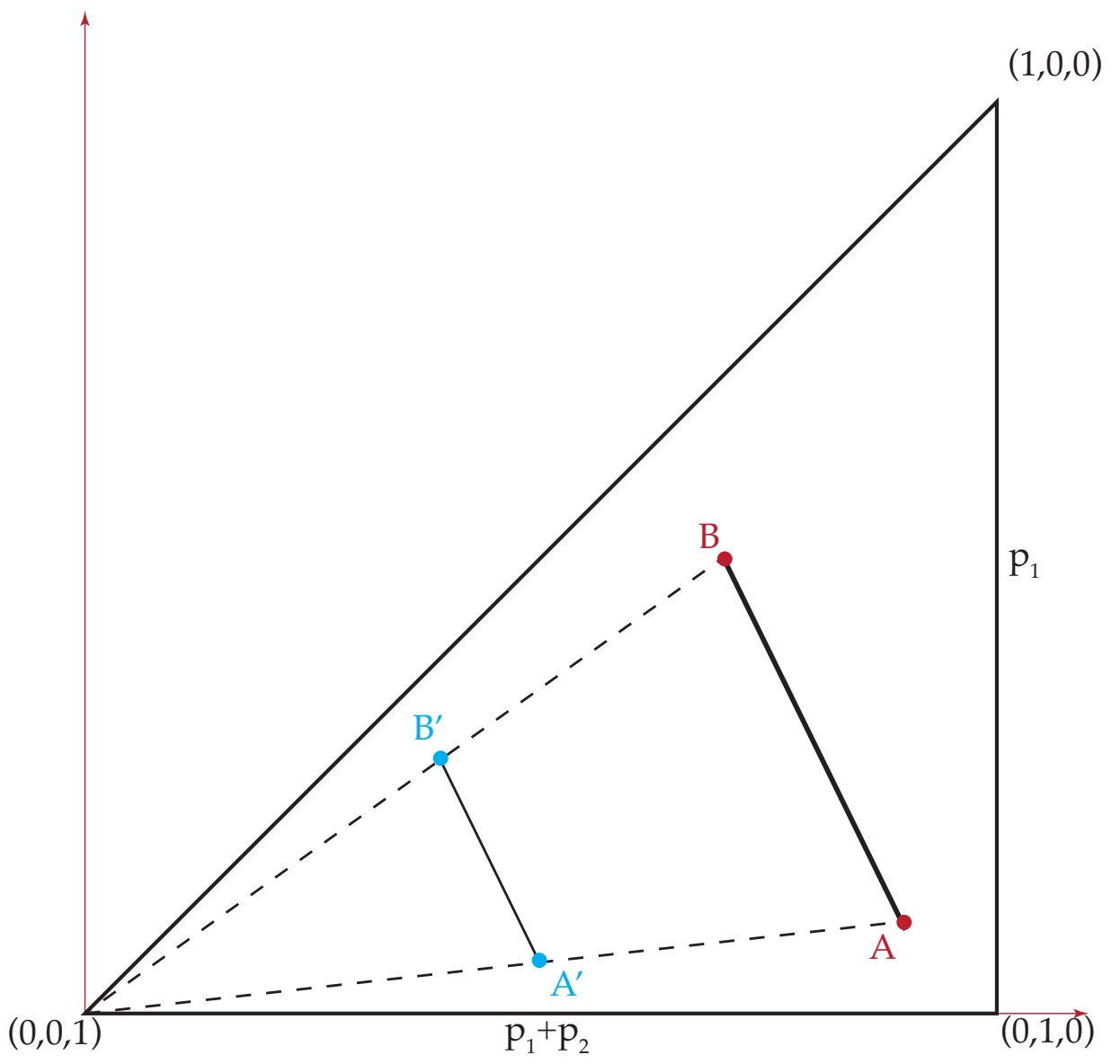




\subsection{Empirically disentangling independence, betweenness, and homotheticity}

Proposition 1 has empirical value because it provides an indirect mechanism for testing betweenness: If preferences violate independence, but satisfy homotheticity, it is because betweenness is violated. Panels (a) and (b) of Figure 4 provide an illustration of how this can occur. These figures depict two, three-alternative choice-situations in the simplex. The set of alternatives on the red line-segment, $\{A, B, M\}$, includes a lottery along the lower edge of the simplex $(B)$, a lottery along the hypotenuse $(A)$, and an alternative on the interior of the simplex that is a mixture of $A$ and $B$ (i.e. $M=\alpha A+(1-\alpha) B$, where $\alpha$ is in the unit interval). The set of alternatives on the blue line-segment, $\left\{A^{\prime}, B^{\prime}, M^{\prime}\right\}$, has an identical setup. Note that these two line-segments intersect on the interior of the simplex. It is for this reason that we refer to this setting as X-type choice-situations.

To highlight how X-type choice-situations can detect violations of independence that cannot be explained by betweenness, imagine that the alternatives $M$ and $M^{\prime}$ were selected from the red and blue line-segments, respectively. Taking a weak-preference interpretation (i.e. $\succsim)$, these choices can be rationalized with a betweenness representation only if indifference curves lie precisely on top of the red and blue line-segments. As depicted in Panel (a) of Figure 4, this knife-edge rationalization indicates that these betweenness-satisfying indifference curves would need to cross, a clear violation of transitivity. Thus, betweenness-satisfying preferences cannot accommodate the choices $M$ and $M^{\prime}$ in this setting. That betweenness cannot accommodate these choices also means that they are a violation of independence. On the other hand, the choices $M$ and $M^{\prime}$ can be accommodated by homothetic preferences. Panel (b) of Figure 4 depicts a pair of homothetic indifference curves that rationalize the choices $M$ and $M^{\prime}$.

To illustrate how failures of independence can be attributed to homotheticity but not betweenness, Panels (c) and (d) of Figure 4 depict what we call U-type choice-situations. U-type choicesituations are similar to X-type choice-situations in that there are three alternatives available on each line segment, two along the border of the simplex and a 50/50 mixture in the interior. The line segments in U-type choice-situations must have different slopes, and they cannot intersect.

Using a weak preference interpretation (i.e. $\succsim)$, any pattern of choices in U-type choice- 
situations can be rationalized with a betweenness representation. The most extreme example here involves a pattern of choices that includes one, or both, of the mixture alternatives $\left(M\right.$ or $\left.M^{\prime}\right)$ - any pattern of choices that includes one or more of these alternatives requires a betweennesssatisfying indifference curve to lie precisely on top of the respective line-segment. While such knife-edge rationalizations seem unlikely, they are permissible with the weak preference interpretation we follow here. In contrast to betweenness, the parallelism requirement of homotheticity puts much more structure on choices: If $B$ is selected from $\{A, B, M\}$ then $A^{\prime}, B^{\prime}$, or $M^{\prime}$ are permissible choices under homothetic preferences. If $M$ is selected only $A^{\prime}$ and $M^{\prime}$ are permissible. If $A$ is selected, only $A^{\prime}$ is permissible, a case illustrated in Panel (d) of Figure 4.

These examples illustrate that X-type and U-type choice-situations have distinctive capabilities for detecting failures of independence attributable to failures of betweenness or failures of homotheticity. Given our interest in this regard, it seems natural to contemplate a broader experimental design that includes both X-type and U-type settings. In the next section we discuss one such design. 
Figure 4: X-type and U-type choice-situations

(a) X-type choice-situations - Betweenness preferences cannot rationalize the choices $M$ and $M^{\prime}$

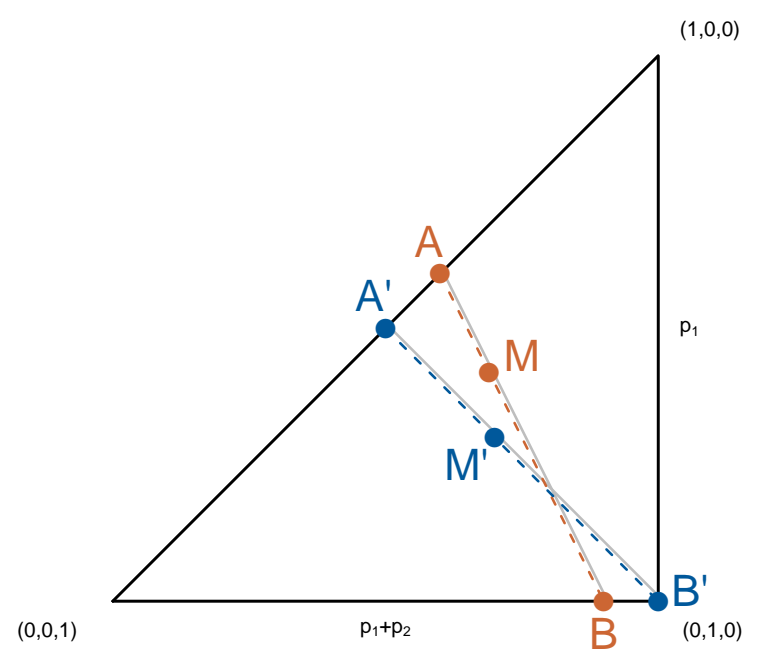

(c) U-type choice-situations - Betweenness preferences can rationalize any pattern of choices

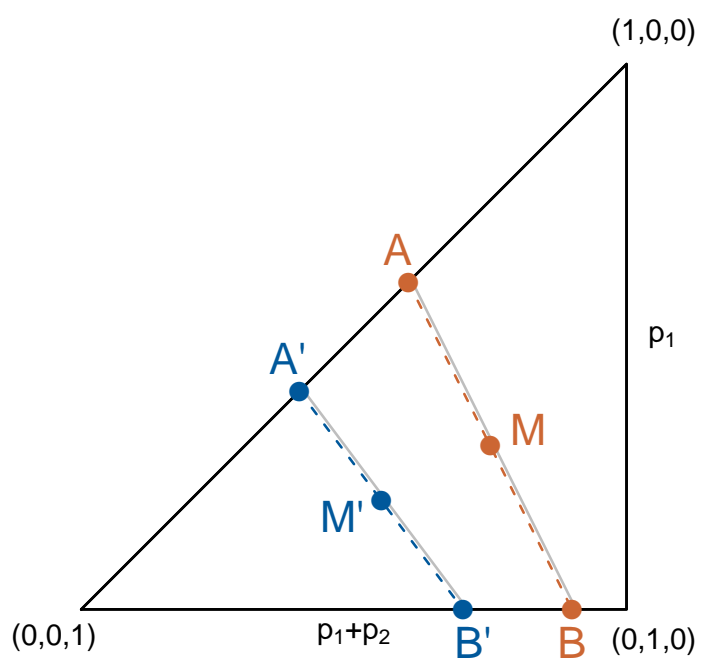

(b) X-type choice-situations - Homothetic preferences can rationalize the choices $M$ and $M^{\prime}$

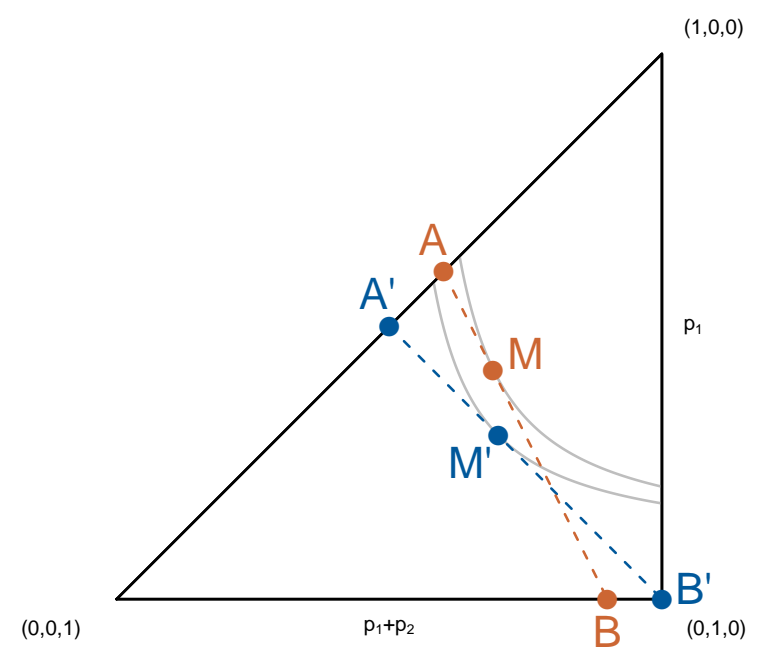

(d) U-type choice-situations - Homothetic preferences cannot rationalize the choices $B$ and $A^{\prime}$

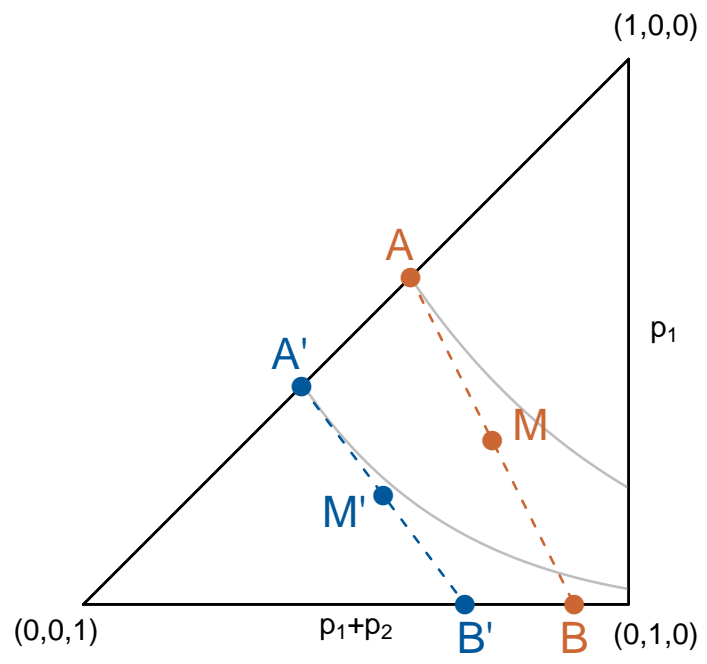




\section{Experimental Methods}

The choice-situations in our experiment were designed as a set of overlapping X-type and U-type choice-situations (see section 1.6 for definitions) to allow for direct assessments of independence and homotheticity. Figure 5 depicts, in the probability triangle, the line-segments, and lotteries, used to construct this set of overlapping experiments - the line-segments are blue and the lotteries are red points on the line-segments. Each line-segment contains nine red points because each choice-situation in our experiment contained nine lotteries.

\subsection{Experimental design}

As can be seen in Figure 5, none of the red points are on an edge or a vertex in the probability triangle - every lottery available in our experiment always had strictly positive probability mass assigned to each of the three possible outcomes. For this initial analysis of homotheticity we felt it prudent to exclude border and vertex lotteries so as to mitigate any potential behavioral distortions related to certainty effects or differing numbers of possible outcomes.

The horizontal line-segments in Figure 5 were included to permit direct assessments of monotonicity in lottery choices - movements from left, to right, along one of these horizontal line-segments keeps the probability of receiving the best outcome constant while increasing the probability of the intermediate outcome and decreasing the probability of the worst outcome. Preferences that conform to monotonicity will welcome this tradeoff and select the right-most alternative. As we document in Appendix A.2, about 90\% of participants made choices perfectly consistent with monotonicity by selecting the right-most alternative. 4

Three previous experimental studies are closely related to the design of our experiment: Hey and Orme (1994), Camerer and Ho (1994), and Choi, Fisman, Gale, and Kariv (2007). We discuss how our study relates to, and is distinct from, each of these reports.

Hey and Orme (1994) is the closest analogue to our study in terms of experimental design. The experimental design used by Hey and Orme (1994) involved a series of two-alternative

\footnotetext{
${ }^{4}$ Appendix A.5 contains specific details about our experimental design including an exhaustive listings of all the lotteries available in each choice-situation, general construction details, and the instructional packet that was given to participants.
} 
choice-situations that, when plotted in a probability triangle, represent a set of intersecting and non-intersecting line-segments that included alternatives on the strict interior, edges, and vertices of the simplex. Our experimental design is differentiated by the inclusion of nine alternatives in each choice-situation, each on the strict interior of the simplex, and a systematic arrangement of overlapping X-type and U-type choice-situations. The Hey and Orme (1994) experiment was designed to maximize the power of discriminating between many different types of risky-choice models, whereas our experiment was designed so as to maximize the power of detecting failures of just independence and homotheticity.

Another way in which our study differs from Hey and Orme (1994) is analytical approach. Hey and Orme (1994) deploy a "top-down" approach to assessing axiomatic criteria like independence and betweenness - they examined the statistical goodness-of-fit for different model specifications assuming an additive error structure. In contrast, our study takes a "bottom-up" approach and assesses whether choices are consistent with necessary and sufficient conditions for individual axioms.

Camerer and Ho (1994) also used experimental data to examine whether choices could be consistent with betweenness. They did this by eliciting strict preferences (i.e. $\succ$ ) in their binary choice-situations, all of which involved pairwise combinations of lotteries on the same linesegment. Our experimental design is differentiated by the inclusion of line-segments that have varying slopes, each of which contains nine alternatives. Our analysis is further differentiated through the elicitation of only weak preferences ${ }^{5}$ and the use of revealed preference methods.

The study by Choi, Fisman, Gale, and Kariv (2007) also used revealed preference methods to study risky-choices. However, Choi, Fisman, Gale, and Kariv (2007) focused on preferences for outcomes under a fixed probability distribution. This focus is a natural complement to our study. We focus on preferences for probabilities over a fixed set of outcomes, also using revealed preference methods.

\footnotetext{
In their binary choice-situations, Camerer and Ho (1994) asked participants to report strict preferences or indifference for lotteries.
} 
Figure 5: The line segments and lotteries used in our experiment depicted in the probability triangle

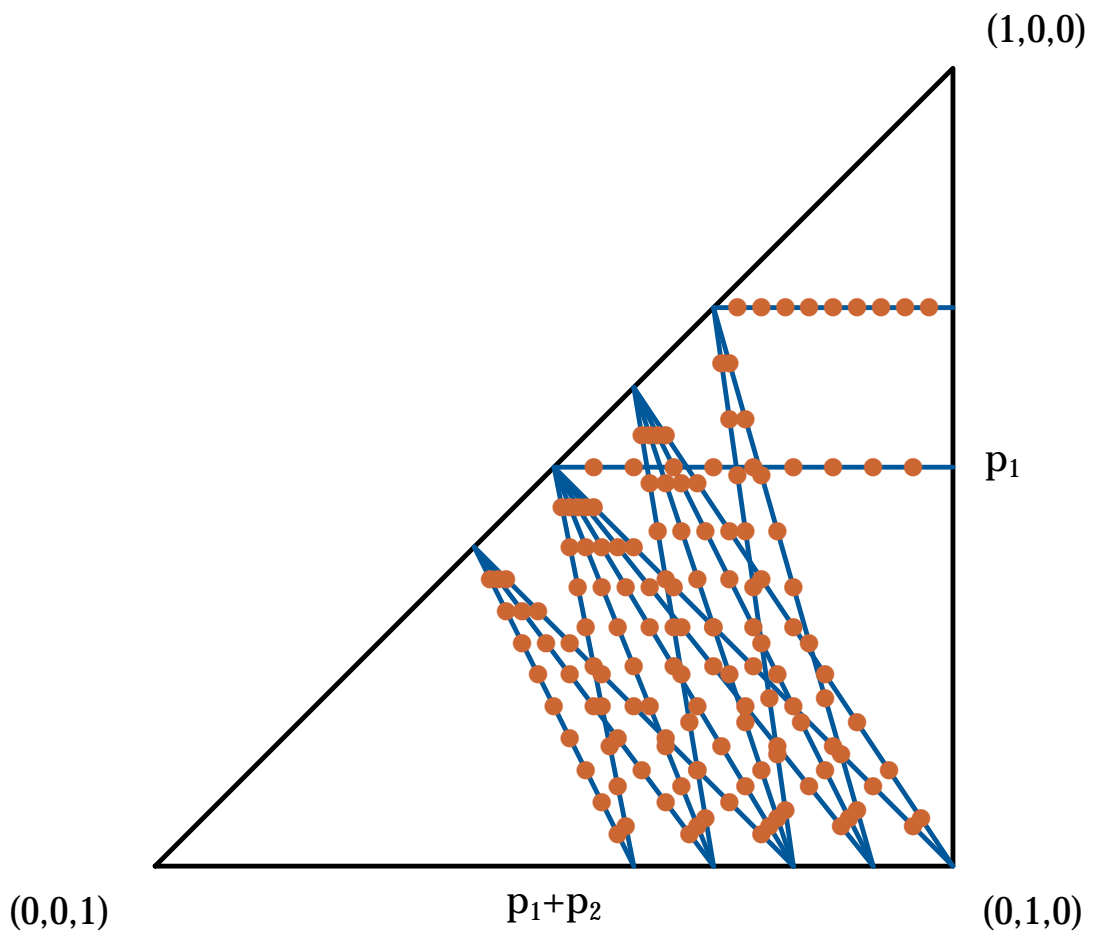




\subsection{Choice elicitation software}

Each choice-situation in our experiment consisted of nine lotteries. Each of nine lotteries had three possible outcomes. In one half of the choice-situations every lottery available had possible outcomes of Swiss Francs (CHF) $\{40,20,0\}$. In the other half every lottery available had possible outcomes of CHF $\{60,40,20\}$. At the time of the experiment 1 CHF was equivalent to 1.05 USD. The two screenshots from our elicitation software in Figure 6 illustrates that all lotteries with outcomes of CHF $\{40,20,0\}$ were presented visually using a blue color scheme. All lotteries with outcomes of CHF $\{60,40,20\}$ were presented visually using a yellow color scheme. Where appropriate, and for brevity, we will henceforth refer to the $\operatorname{CHF}\{40,20,0\}$ choice-environment as low, and the CHF $\{60,40,20\}$ choice-environment as high.

Figure 6 shows two screenshots from our choice elicitation software, which was programmed using the Psychtoolbox Matlab libraries freely available at http://www.psychtoolbox.org. This elicitation software required only mouse-based input from participants. Placing the mouse cursor over one of the nine thumbnails at the bottom of the screen, thumbnails depicting each of the nine lotteries available in the choice-situation, highlighted the thumbnail with a green surround. Once highlighted, the details for the lottery depicted in that thumbnail were drawn to the large lottery in the middle of the screen. The large lottery detailed the specific outcome and probability information in text. All lotteries were presented using a vertical bar divided into three differently colored blocks. The color of the blocks corresponded to specific outcome values. The height of each block was proportional to the probability assigned to its associated outcome. The ordering of the blocks was such that the largest outcome was always on the bottom and the smallest outcome was always on top. The participant's task was to choose one of the nine lotteries and we interpret their choice as weakly revealed preferred to the other alternatives. To make a choice, participants had to click on one of the thumbnail images. After clicking, the highlighted box was locked and "Confirm" and "Cancel" boxes appeared on screen. Clicking the "Confirm" box advanced the software to the next choice-situation while a click inside the "Cancel" box unlocked the highlighter, thereby permitting a different choice. 


\subsection{Procedures}

All experimental session were conducted between 1 p.m. and 5 p.m. during one week in the 2013 Spring semester at the University of Zurich/ETH Zurich (UZH). Participants provided informed consent consistent with a procedure approved by the UZH Institutional Review Board and all sessions occurred at the ETH Decision Lab.

After participants entered the experimental laboratory and were seated at their randomly assigned carrel, instructions were read by the experimenter over the in-lab public-address system while participants followed along. After the instructional phase participants engaged in a brief practice/exploratory session using the computer at their carrel to become familiar with the elicitation software. After participants completed the choice experiment the software randomly selected one choice-situation to count for actual payment. The software displayed the choicesituation selected by the computer and the lottery the participant selected. An experimenter wrote down the details of this payment lottery on a sheet of paper with the participant's randomly assigned ID number on it. Participants then initialed this paper which was taken to the office while participants completed a brief survey. Once the survey was completed participants were queued one-by-one to a separate room in which they rolled two, 1o-sided dice to resolve the uncertainty of their lottery (for details, see the instructions at the end of the Appendix). 
Figure 6: Screenshots from the experimental software showing the same line-segment and highlighted alternative in the low and high choice environments

(a) The low choice environment with possible outcomes Swiss Francs (CHF) $\{40,20,0\}$

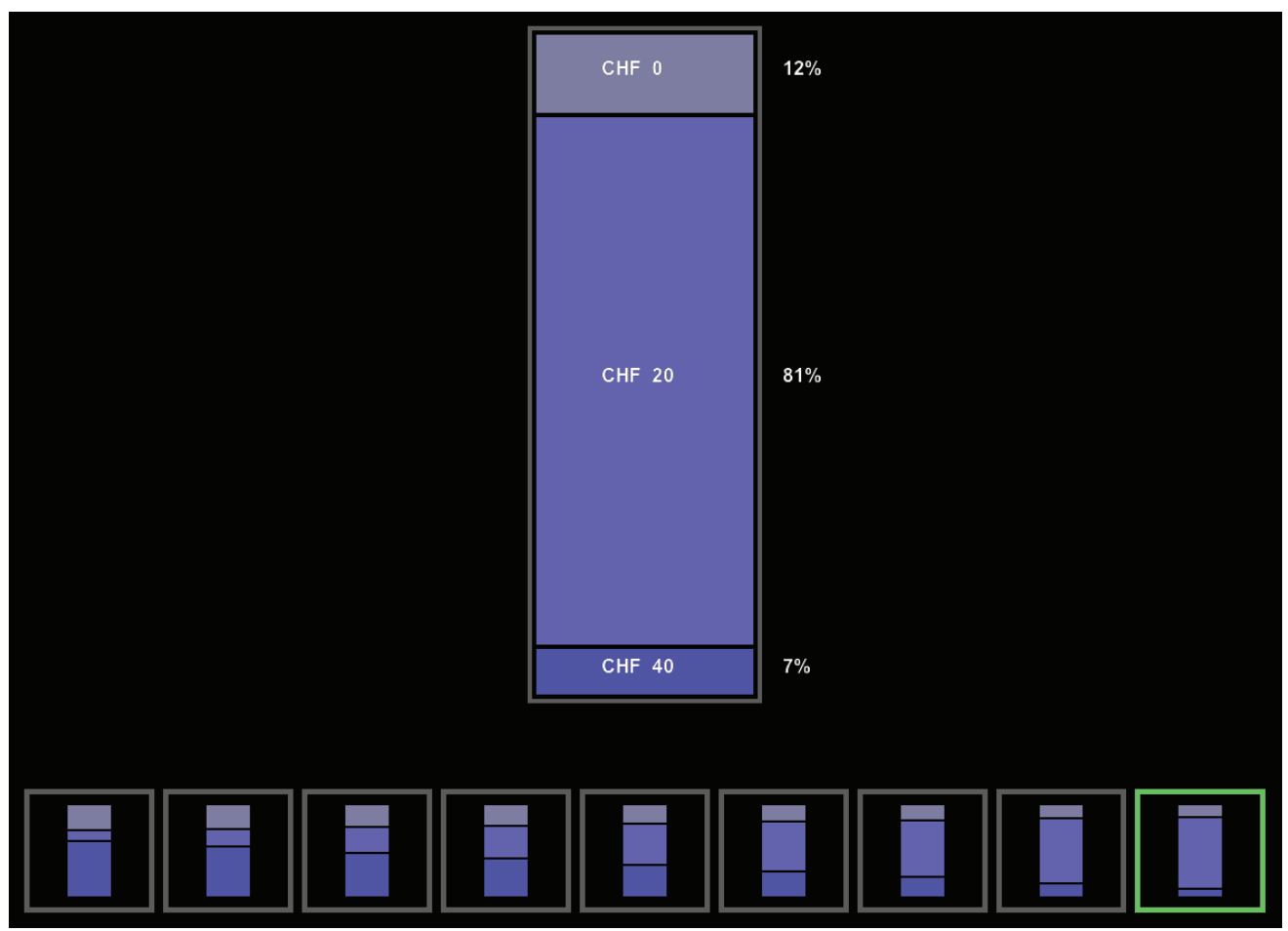

(b) The high choice environment with possible outcomes Swiss Francs (CHF) $\{60,40,20\}$

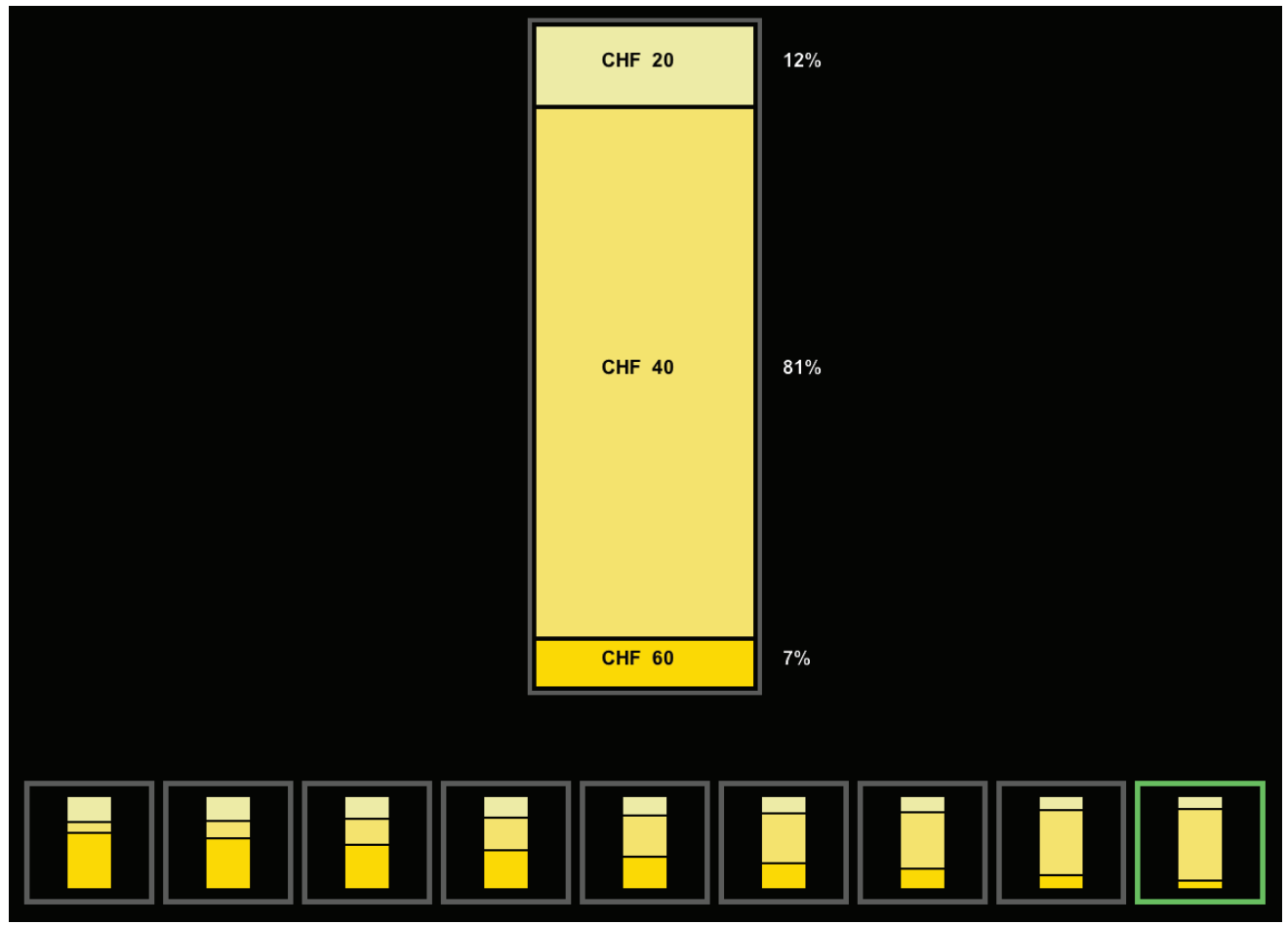




\section{Analysis}

Proposition 1 embeds an indirect test of the betweenness axiom - if preferences satisfy homotheticity, but fail independence, then betweenness is violated. In order to carry out this indirect test of betweenness, homotheticity and independence need to be tested directly. This section details how we carried out these direct tests.

Let $A^{i}=\left(p_{1}^{i}, p_{2}^{i}, p_{3}^{i}\right)$ represent the lottery that was selected from line-segment $i$ in our experiment. To make it easier to conduct our analyses, we convert these three-tuples into two-tuples:

$$
A^{i}=\left(p_{1}^{i}, p_{2}^{i}, p_{3}^{i}\right) \rightarrow\left(p_{1}^{i}+p_{2}^{i}, p_{1}^{i}\right)=\left(x_{1}^{i}, x_{2}^{i}\right)=\mathbf{x}^{i} \in \Delta_{\mathbf{x}}
$$

This transformation moves three-outcome lotteries into a two-dimensional coordinate system that has its origin at our homothetic center, the worst outcome. We interpret these two-tuples, $\left(x_{1}^{i}, x_{2}^{i}\right)$, as demand vectors relative to the homothetic center of the worst outcome, an interpretation that will simplify our direct tests of homotheticity and independence. We use the notation $\Delta_{\mathbf{x}}$ to denote the set of all such two-tuples that are valid lotteries in this setting. Formally, $\Delta_{\mathbf{x}} \equiv\{\mathbf{x} \in$ $\left.[0,1]^{2}: x_{2} \leq x_{1}\right\}$

Next, we formalize the line-segments used to construct choice-situations in our experiment as linear budgets, also relative to the homothetic center.

Definition. Given a vector of strictly positive prices $q^{i}=\left(q_{1}^{i}, q_{2}^{i}\right)$, and wealth $w^{i}>0$, a probability budget is the collection of points $P B_{\boldsymbol{q}^{i}, w^{i}} \equiv\left\{\boldsymbol{x} \in \Delta_{\boldsymbol{x}} \mid \boldsymbol{q}^{i} \cdot \boldsymbol{x}=w^{i}\right\}$.

The combination of prices $\left(\mathbf{q}^{i}\right)$ and demands $\left(\mathbf{x}^{i}\right)$, both relative to the homothetic center of the worst outcome, make it easy to adapt revealed preference tests in this setting. And, our notation for prices and demands also lets us closely mirror the notation of Varian (1983).

We analyze choices from 110 participants in our experiment. As we detail in appendices A.2 and A.3, these 110 participants (i) made choices that are consistent with our direct tests of monotonicity (i.e. they selected the right-most alternative in each of the horizontal line-segments set up as direct tests of monotonicity) and (ii) made choices that conform to the generalized axiom of revealed preference (GARP). For the linear budget setting in our experiment, Afriat's 
theorem guarantees that choices conforming to GARP can be modeled with a preference relation that is complete, transitive, continuous, and monotonic (Varian, 1982).

\subsection{Directly testing homotheticity}

Our first direct test is for homotheticity. We apply Varian (1983)'s homothetic axiom of revealed preference (HARP) to the prices $\left(\mathbf{q}^{i}\right)$ and demands $\left(\mathbf{x}^{i}\right)$ in our experimental data. The homothetic axiom is a necessary and sufficient condition for a homothetic representation. Denote the 14 choices made from the probability budgets with strictly positive prices, in either the low or high choice-environment, as $\left(\mathbf{x}^{1}, \ldots, \mathbf{x}^{14}\right)$ and the prices as $\left(\mathbf{q}^{1}, \ldots, \mathbf{q}^{14}\right)$. As documented by Varian (1983), the homothetic axiom of revealed preference requires the following inequality to hold:

$$
\left(\mathbf{q}^{i} \cdot \mathbf{x}^{j}\right)\left(\mathbf{q}^{j} \cdot \mathbf{x}^{k}\right) \ldots\left(\mathbf{q}^{m} \cdot \mathbf{x}^{i}\right) \leq w^{i} w^{j} \ldots w^{m}
$$

for all of the possible combinations of indices $i, j, k \ldots, m$. Normalizing prices by the wealth at each observation, such that $\mathbf{q}^{i} \cdot \mathbf{x}^{i}=1$, then taking logs, the homothetic axiom can be restated as the following condition:

$$
\log \left(\mathbf{q}^{i} \cdot \mathbf{x}^{j}\right)+\log \left(\mathbf{q}^{j} \cdot \mathbf{x}^{k}\right)+\cdots+\log \left(\mathbf{q}^{m} \cdot \mathbf{x}^{i}\right) \geq 0
$$

Varian (1983) provides an algorithm for testing this condition using a computer.

\subsubsection{Algorithm}

To test for violations of the homothetic axiom, Varian (1983) provides a graph-theoretic interpretation for Equation 4 - the "cost" of moving from node $i$ to itself cannot be made less than zero. As Varian demonstrates, this condition is easily tested by applying Warshall's algorithm for finding the lowest cost path in a revealed preference graph. Warshall's algorithm first requires the construction of a matrix $C$ that represents the costs of moving from vertex $i$ to vertex $j$, such that $c_{i j}=\mathbf{q}^{i} \cdot \mathbf{x}^{j}$ (note that matrix $C$ is the directly revealed preferred graph). Then a matrix of zeros is initialized $(\bar{C})$. Warshall's algorithm guarantees that the lowest cost path in a graph can 
be found by searching independently over the indexes $i, j, k=1, \ldots, 14$, and replacing the $i j^{\text {th }}$ entry in $\bar{C}$ with $c_{i k}+c_{k j}$ whenever the following inequality holds:

$$
c_{i k}+c_{k j} \leq c_{i j}
$$

If any of the entries on the diagonal of $\bar{C}$ are less than zero, it means that there is a sequence of observations such that the cost of moving from node $i$ to itself can be made less than zero. As stated in Equation 4, this means the homothetic axiom is violated and therefore choices cannot be rationalized with homothetic preferences.

\subsubsection{Results}

Of the 110 participants in our experiment, only nine made choices that were perfectly consistent with the homothetic axiom in both the low and high choice-environments. While consistency with any axiom is a binary consideration - choices are either perfectly consistent with the axiom or they are not - such binary considerations do not provide a sense of how badly homotheticity was violated. To measure how badly homotheticity was violated we use the Houtman-Maks index (Houtman and Maks, 2007).

Houtman-Maks represents the largest subset of choices that are consistent with the homothetic axiom of revealed preference (HARP). Traditionally, the Houtman-Maks index is used to demarcate the largest subset of choices that are consistent with the generalized axiom of revealed preference (GARP). Here, we adapt the logic of the traditional Houtman-Maks to the homothetic axiom. And we adopt HM-HARP as shorthand. So, for the nine participants who made choices perfectly consistent with HARP, their HM-HARPs would be 14 in both the low and high choiceenvironments - There were 14 choices made in each environment and all 14 of those choices were consistent with HARP. If, however, a participant's full set of 14 choices was not consistent with HARP but, by removing just one choice such that a subset of size 13 was consistent with HARP, then this hypothetical subject's HM-HARP would be 13. To find the HM-HARP for each subject we used a brute-force approach - If HARP was violated for the full set of 14 choices we checked all subsets of size 13. If a subset of size 13 was found to be consistent with HARP we stopped 
looking and HM-HARP was 13. If no subset of size 13 was consistent with HARP, we checked all subsets of size 12. And we continued on in such a manner until a subset of choices was found to be consistent with HARP.

Table 1 shows the number of participants, at each HM-HARP value, by choice-environment. The bottom row in Table 1 shows the marginal distribution of HM-HARPs in the low choiceenvironment (i.e. the possible outcomes from the lotteries were $\mathrm{CHF}\{40,20,0\}$ ). The right-most column in Table 1 shows the marginal distribution for the high choice-environment (i.e. the possible outcomes from the lotteries were $\operatorname{CHF}\{60,40,20\})$. For example, the entry on the row labeled 12 and column labeled 11 indicates that 2 participants had HM-HARPs of 12 and 11 in the high and low choice environments, respectively.

Without critical-values, HM-HARPs provide no context as to whether choices can be considered consistent with, or inconsistent with, homotheticity. To draw an analogy with classical statistics - $\mathrm{t}$-values arising from a comparison of means are useless without critical values. As is the case for critical t-values, what is needed for HM-HARPs are critical values that serve as a cutoff for demarcating participants whose choices can be considered consistent with, or inconsistent with, homotheticity. As in previous work, our construction of critical values derives from a Monte Carlo analysis of a large number of synthetic experimental participants who have a uniform random choice rule. ${ }^{6}$ Table 6 in Appendix A.4 provides a sampling distribution of HM-HARPs for 15,000 synthetics with an independent, uniform random choice rule.

In Table 1 , the number of subjects whose HM-HARPs exceed a 95\% critical value from this Monte Carlo analysis are indicated with a dagger $(\dagger)$. A total of 74 participants met or exceeded this $95 \%$ critical value. These 74 participants represents $67 \%$ (approximately $2 / 3$ 's) of the 110 participant choices we analyze here. We refer to the approximately $2 / 3$ 's of participants whose HM-HARPs met or exceeded the $95 \%$ critical value as having preferences that are consistent with homotheticity.

\footnotetext{
${ }^{6}$ In the case of the homothetic axiom, see Heufer (2012). This method is also used for assessments of the generalized axiom. See, for example, Harbaugh, Krause, and Berry (2001) and Burghart, Glimcher, and Lazzaro (2013)
} 
Table 1: Approximately 2/3's of participants make choices consistent with homotheticity because their HM-HARPs meet or exceed a 95\% critical value.

\begin{tabular}{|c|c|c|c|c|c|c|c|c|c|c|c|}
\hline & \multicolumn{11}{|c|}{ Row/Column Headings are HM-HARPs } \\
\hline & & \multicolumn{9}{|c|}{\begin{tabular}{|l} 
Low \\
\end{tabular}} & \multirow[b]{2}{*}{ Total } \\
\hline & & 14 & 13 & 12 & 11 & 10 & 9 & 8 & 7 & 6 & \\
\hline \multirow{9}{*}{ High } & 14 & $9^{+}$ & $22^{t}$ & $3^{t}$ & $\mathrm{O}$ & $\mathrm{O}$ & $\mathrm{O}$ & $\mathrm{O}$ & $\mathrm{O}$ & $\mathrm{O}$ & 14 \\
\hline & 13 & $1^{\dagger}$ & $5^{\dagger}$ & $1^{\dagger}$ & o & $1^{\dagger}$ & $\mathrm{O}$ & o & o & o & 8 \\
\hline & 12 & $\mathrm{O}$ & $5^{\dagger}$ & $6^{\dagger}$ & $2^{\dagger}$ & $2^{\dagger}$ & 1 & o & $\mathrm{O}$ & $\mathrm{O}$ & 16 \\
\hline & 11 & $2^{\dagger}$ & $2^{\dagger}$ & $6^{+}$ & $3^{\dagger}$ & $4^{\dagger}$ & 3 & 1 & $\mathrm{O}$ & $\mathrm{O}$ & 21 \\
\hline & 10 & $\mathrm{O}$ & $7^{\dagger}$ & $1^{\dagger}$ & $7^{\dagger}$ & $5^{\dagger}$ & $\mathrm{O}$ & o & $\mathrm{o}$ & $\mathrm{O}$ & 20 \\
\hline & 9 & $\mathrm{o}$ & 1 & $\mathrm{o}$ & 2 & $\mathrm{o}$ & 1 & 3 & 2 & 1 & 10 \\
\hline & 8 & 1 & $\mathrm{o}$ & 1 & 2 & $\mathrm{o}$ & 4 & 2 & $\mathrm{o}$ & $\mathrm{o}$ & 10 \\
\hline & 7 & $\mathrm{o}$ & $\mathrm{o}$ & 2 & 1 & $\mathrm{o}$ & 1 & 1 & 1 & $\mathrm{o}$ & 6 \\
\hline & 6 & $\mathrm{O}$ & $\mathrm{o}$ & $\mathrm{O}$ & 2 & 1 & 1 & 1 & $\mathrm{O}$ & $\mathrm{O}$ & 5 \\
\hline Total & & 13 & 22 & 20 & 19 & 13 & 11 & 8 & 3 & 1 & 110 \\
\hline
\end{tabular}

Notes: A + indicates participant HM-HARPs that exceed a $95 \%$

critical value determined by a Monte Carlo analysis of 15,000

synthetics with a uniform-random choice rule.

\subsection{Directly testing independence}

Our second direct test is for the independence axiom. Preferences that satisfy independence have a geometric interpretation - indifference curves are parallel straight lines in the probability triangle. This graphical interpretation carries over to our analysis of lotteries in a two-coordinate system - indifference curves in $\Delta_{\mathbf{x}}$ that are consistent with independence must also be parallel straight lines. In our two-coordinate system, parallel straight line indifference curves are equivalent to a perfect substitutes representation for $x_{1}$ and $x_{2}$. And our direct test of independence draws on the properties of a perfect substitutes representation in this setting - given a vector of demands arising at some prices, if the steepness of the probability budget changes, then demands must move in a predictable way.

Consider, for example, the three budgets depicted in Figure 7. Suppose that the choice from the middle budget is the alternative closest to the horizontal leg of the triangle. Letting the orange dot signify the choice from the middle budget, any independence-satisfying indifference curve that can rationalize this choice must lie in the green shaded area. And because independencesatisfying indifference curves must be parallel, the choice from a flatter budget, like the left-most 
Figure 7: Predicting choices when assuming adherence to the independence axiom

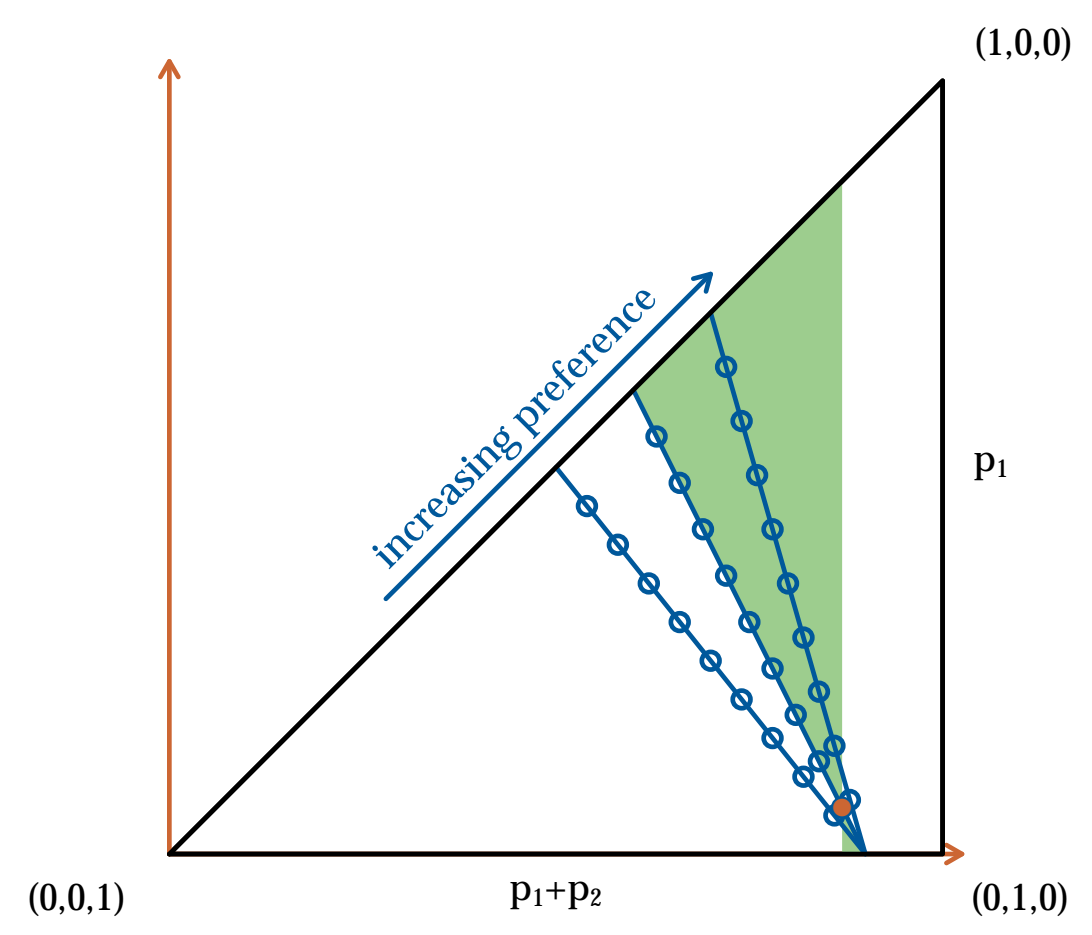

budget in Figure 7, can be predicted: it must also be the alternative closest to the horizontal leg of the triangle. When a budget is steeper than the original budget, however, no such prediction can be made. Of course, if the choice from the middle budget was the lottery closest to the hypotenuse, then the reverse predictions can be made. Choices from steeper budgets would be predicted to be from the hypotenuse, while no prediction about choices would be possible from flatter budgets. And finally, if the original choice from the middle budget was from the strict interior of the budget, any independence-satisfying indifference curve would need to lie exactly on the budget. Because of parallelism, choices from any flatter budget would be predicted to be the lottery closest to the horizontal leg. And choices from steeper budgets would be predicted to be the lottery closest to the hypotenuse. 


\subsubsection{Creating predictions to directly test independence}

To formalize, and exhaustively catalog these independence-related predictions, we need to compare the steepness of the $i^{\text {th }}$ budget, denoted by the ratio of its prices $\left(\frac{q_{1}}{q_{2}}\right)^{i}$, to the steepness of the $j^{\text {th }}$ budget, denoted by the ratio of its prices $\left(\frac{q_{1}}{q_{2}}\right)^{j}$. We say:

1. The $j^{\text {th }}$ budget is flatter than the $i^{\text {th }}$ budget whenever $\left(\frac{q_{1}}{q_{2}}\right)^{j}<\left(\frac{q_{1}}{q_{2}}\right)^{i}$

2. The $j^{\text {th }}$ budget is steeper than the $i^{\text {th }}$ budget whenever $\left(\frac{q_{1}}{q_{2}}\right)^{j}>\left(\frac{q_{1}}{q_{2}}\right)^{i}$

Based on the choices made from the $i^{\text {th }}$ budget, we can use these steepness comparisons to make an exhaustive list of predictions. about all the other $j^{\text {th }}$ budgets:

- Case 1: The choice from probability budget $i$ is at the "corner" closest to the horizontal-axis.

Denote this corner alternative by $\lfloor\mathbf{x}\rfloor^{i}$

$$
\mathbf{x}^{i}=\lfloor\mathbf{x}\rfloor^{i} \Rightarrow \begin{cases}\mathbf{x}^{j}=\lfloor\mathbf{x}\rfloor^{j} & \text { when }\left(\frac{q_{1}}{q_{2}}\right)^{j}<\left(\frac{q_{1}}{q_{2}}\right)^{i} \\ \text { no prediction } & \text { when }\left(\frac{q_{1}}{q_{2}}\right)^{j}>\left(\frac{q_{1}}{q_{2}}\right)^{i} \\ \text { no prediction } & \text { when }\left(\frac{q_{1}}{q_{2}}\right)^{j}=\left(\frac{q_{1}}{q_{2}}\right)^{i}\end{cases}
$$

- Case 2: The choice from probability budget $i$ is at the "corner" closest to the hypotenuse.

Denote this corner alternative by $\lceil\mathbf{x}\rceil^{i}$

$$
\mathbf{x}^{i}=\lceil\mathbf{x}\rceil^{i} \Rightarrow \begin{cases}\text { no prediction } & \text { when }\left(\frac{q_{1}}{q_{2}}\right)^{j}<\left(\frac{q_{1}}{q_{2}}\right)^{i} \\ \mathbf{x}^{j}=\lceil\mathbf{x}\rceil^{j} & \text { when }\left(\frac{q_{1}}{q_{2}}\right)^{j}>\left(\frac{q_{1}}{q_{2}}\right)^{i} \\ \text { no prediction } & \text { when }\left(\frac{q_{1}}{q_{2}}\right)^{j}=\left(\frac{q_{1}}{q_{2}}\right)^{i}\end{cases}
$$

- Case 3: The choice from probability budget $i$ is on the "interior" (i.e. not Case 1 or Case 2). 
Denote these alternatives by $[\mathbf{x}]^{i}$

$$
\mathbf{x}^{i} \in[\mathbf{x}]^{i} \Rightarrow \begin{cases}\mathbf{x}^{j}=\lfloor\mathbf{x}\rfloor^{j} & \text { when }\left(\frac{q_{1}}{q_{2}}\right)^{j}<\left(\frac{q_{1}}{q_{2}}\right)^{i} \\ \mathbf{x}^{j}=\lceil\mathbf{x}\rceil^{j} & \text { when }\left(\frac{q_{1}}{q_{2}}\right)^{j}>\left(\frac{q_{1}}{q_{2}}\right)^{i} \\ \text { no prediction } & \text { when }\left(\frac{q_{1}}{q_{2}}\right)^{j}=\left(\frac{q_{1}}{q_{2}}\right)^{i}\end{cases}
$$

We say choices are consistent with an independence representation if two conditions are met:

- Condition 1: Predictions are internally consistent.

- Condition 2: Choices are consistent with the predictions.

The first condition requires that two choices arising from distinct budgets do not generate conflicting predictions regarding a third choice-situation. The second condition is more natural and simply requires that choices are consistent with the set of (internally consistent) predictions.

\subsubsection{Algorithm}

Algorithmically, we test these condition using a computer. We begin by constructing three square matrices, $\lfloor D\rfloor,\lceil D\rceil$, and $[D]$, with entries defined, respectively:

$$
\begin{aligned}
& \lfloor d\rfloor_{i j}= \begin{cases}-1 & \text { if } \mathbf{x}^{i}=\lfloor\mathbf{x}\rfloor^{i} \text { and }\left(\frac{q_{1}}{q_{2}}\right)^{j}<\left(\frac{q_{1}}{q_{2}}\right)^{i} \\
0 & \text { otherwise }\end{cases} \\
& \lceil d\rceil i j= \begin{cases}+1 & \text { if } \mathbf{x}^{i}=\lceil\mathbf{x}\rceil^{i} \text { and }\left(\frac{q_{1}}{q_{2}}\right)^{j}>\left(\frac{q_{1}}{q_{2}}\right)^{i} \\
0 & \text { otherwise }\end{cases} \\
& {[d]_{i j}= \begin{cases}-1 & \text { if } \mathbf{x}^{i} \in[\mathbf{x}]^{i} \text { and }\left(\frac{q_{1}}{q_{2}}\right)^{j}<\left(\frac{q_{1}}{q_{2}}\right)^{i} \\
+1 & \text { if } \mathbf{x}^{i} \in[\mathbf{x}]^{i} \text { and }\left(\frac{q_{1}}{q_{2}}\right)^{j}>\left(\frac{q_{1}}{q_{2}}\right)^{i} \\
0 & \text { otherwise }\end{cases} }
\end{aligned}
$$


Because $[D]$ is the only matrix that has both +1 and -1 entries (i.e. only when $\mathbf{x}^{i} \in[\mathbf{x}]^{i}$ can we get predictions of both $\lfloor\mathbf{x}\rfloor^{j}$ or $\lceil\mathbf{x}\rceil^{j}$ ) we first check it for internal consistency of its predictions. We construct two row vectors $\operatorname{Max}[D]$ and $\operatorname{Min}[D]$ where

$$
\begin{aligned}
\operatorname{Max}[D] & =\left[\operatorname{Max}\left\{[d]_{.1}, 0\right\}, \operatorname{Max}\left\{[d]_{.2}, 0\right\}, \ldots, \operatorname{Max}\left\{[d]_{{ }_{J},} 0\right\}\right] \\
\operatorname{Min}[D] & =\left[\operatorname{Min}\left\{[d]_{.1}, 0\right\}, \operatorname{Min}\left\{[d]_{.2}, 0\right\}, \ldots, \operatorname{Min}\left\{[d]_{{ }_{J},}, 0\right\}\right]
\end{aligned}
$$

where $[d]_{. j}$ indicates the collection of entries in the $j^{\text {th }}$ column of $[D]$. Notice that $\operatorname{Max}[d]_{j} *$ $\operatorname{Min}[d]_{j} \geq 0, \forall j=1, \ldots, J$ if and only if predictions are internally consistent in $[D]$.

Conditional on $[D]$ exhibiting internal prediction consistency, all that remains is to verify that all three of the following conditions hold:

$$
\begin{array}{r}
\operatorname{Max}\lceil d\rceil_{j} \cdot \operatorname{Min}\lfloor d]_{j} \geq 0, \forall j=1, \ldots, J \\
\operatorname{Max}\lceil d]_{j} \cdot \operatorname{Min}[d]_{j} \geq 0, \forall j=1, \ldots, J \\
\operatorname{Max}[d]_{j} \cdot \operatorname{Min}\lfloor d]_{j} \geq 0, \forall j=1, \ldots, J
\end{array}
$$

where

$$
\begin{aligned}
& \operatorname{Max}\lceil D\rceil=\left[\operatorname{Max}\left\{\lceil d\rceil_{.1}\right\}, \operatorname{Max}\left\{\lceil d\rceil_{.2}\right\}, \ldots, \operatorname{Max}\left\{\lceil d\rceil_{\cdot J}\right\}\right] \\
& \operatorname{Min}\lfloor D\rfloor=\left[\operatorname{Min}\left\{\lfloor d\rfloor_{.1}\right\}, \operatorname{Min}\left\{\lfloor d\rfloor_{.2}\right\}, \ldots, \operatorname{Min}\{\lfloor d\rfloor \cdot J\}\right]
\end{aligned}
$$

If predictions are internally consistent (i.e. choices satisfy condition 1) it is a straightforward exercise to verify that choices are consistent with predictions (i.e. choices satisfy condition 2). If choices satisfy both condition 1 and condition 2 we say they are consistent with an independence representation.

\subsubsection{Results}

Few individuals made all 14 choices that satisfy conditions 1 and 2. However, to assess how badly a set of choices depart from our test of independence we again adapt the logic of the Houtman-Maks index. We label this adapted measure as HM-IA (as in Houtman-Maks Independence Axiom) - HM-IA represents the largest subset of choices that are consistent with the two 
conditions laid out above.

Table 2 shows HM-IA values for the participants in our experiment, in both the low and high choice environments. To find critical values for HM-IA we constructed a sampling distribution for HM-IA from a Monte Carlo analysis. In this simulation, our 15,000 synthetic choosers once again had a uniform random choice rule but were restricted to only those alternatives at the corners of the probability budgets. ${ }^{7}$ As can be seen with the daggers $(\dagger)$ in Table 2, only 37 of our 110 participants' HM-IAs exceeded the $95 \%$ critical value. This represents $34 \%$ of our sample, or approximately $1 / 3$. We refer to the approximately $1 / 3$ of participants whose HM-IAs met or exceeded the $95 \%$ critical value as having preferences that are consistent with independence.

Table 2: Approximately 1/3 of participants make choices consistent with independence because their HM-IAs meet or exceed a $95 \%$ critical value

Row/Column Headings are HM-IAs

\begin{tabular}{|c|c|c|c|c|c|c|c|c|c|c|c|c|c|c|c|}
\hline & \multicolumn{13}{|c|}{ Low } & \multirow{2}{*}{ Total } \\
\hline & & 14 & 13 & 12 & 11 & 10 & 9 & 8 & 7 & 6 & 5 & 4 & 3 & 2 & \\
\hline \multirow{12}{*}{ High } & 14 & $9^{\dagger}$ & $2^{\dagger}$ & $1^{\dagger}$ & $1^{\dagger}$ & $\mathrm{O}$ & $\mathrm{O}$ & $\mathrm{O}$ & $\mathrm{O}$ & $\mathrm{O}$ & $\mathrm{O}$ & $\mathrm{O}$ & $\mathrm{O}$ & $\mathrm{O}$ & 13 \\
\hline & 13 & $1^{\dagger}$ & $5^{\dagger}$ & $2^{\dagger}$ & $\mathrm{O}$ & $\mathrm{O}$ & 1 & $\mathrm{O}$ & $\mathrm{O}$ & $\mathrm{O}$ & $\mathrm{O}$ & $\mathrm{O}$ & $\mathrm{O}$ & $\mathrm{O}$ & 9 \\
\hline & 12 & $\mathrm{O}$ & $3^{t}$ & $2^{\dagger}$ & $1^{\dagger}$ & $\mathrm{O}$ & 1 & $\mathrm{O}$ & 2 & $\mathrm{O}$ & $\mathrm{O}$ & $\mathrm{O}$ & $\mathrm{O}$ & $\mathrm{O}$ & 9 \\
\hline & 11 & $1^{\dagger}$ & $3^{+}$ & $3^{\dagger}$ & $3^{t}$ & 4 & 2 & $\mathrm{O}$ & 1 & $\mathrm{O}$ & $\mathrm{O}$ & $\mathrm{O}$ & $\mathrm{O}$ & $\mathrm{O}$ & 17 \\
\hline & 10 & $\mathrm{O}$ & 2 & 1 & 2 & 2 & 1 & 1 & 2 & $\mathrm{O}$ & 1 & $\mathrm{O}$ & $\mathrm{O}$ & $\mathrm{O}$ & 12 \\
\hline & 9 & $\mathrm{O}$ & 2 & $\mathrm{O}$ & $\mathrm{O}$ & 1 & 3 & $\mathrm{O}$ & 1 & 1 & $\mathrm{O}$ & 1 & $\mathrm{O}$ & 1 & 10 \\
\hline & 8 & $\mathrm{O}$ & $\mathrm{O}$ & 1 & 1 & 3 & 2 & 1 & $\mathrm{O}$ & 1 & 1 & $\mathrm{O}$ & $\mathrm{O}$ & 1 & 11 \\
\hline & 7 & 1 & 1 & 1 & $\mathrm{O}$ & 1 & $\mathrm{O}$ & 1 & $\mathrm{O}$ & $\mathrm{O}$ & $\mathrm{O}$ & $\mathrm{O}$ & $\mathrm{O}$ & $\mathrm{O}$ & 5 \\
\hline & 6 & $\mathrm{O}$ & 0 & $\mathrm{O}$ & 1 & 1 & 1 & 1 & $\mathrm{O}$ & 1 & 1 & 2 & $\mathrm{O}$ & $\mathrm{O}$ & 8 \\
\hline & 5 & $\mathrm{O}$ & $\mathrm{O}$ & $\mathrm{O}$ & $\mathrm{O}$ & $\mathrm{O}$ & $\mathrm{O}$ & $\mathrm{O}$ & 1 & $\mathrm{O}$ & $\mathrm{O}$ & $\mathrm{O}$ & 1 & 1 & 3 \\
\hline & 4 & $\mathrm{O}$ & $\mathrm{O}$ & 2 & $\mathrm{O}$ & $\mathrm{O}$ & 1 & 2 & 1 & 1 & $\mathrm{O}$ & $\mathrm{O}$ & 1 & 2 & 10 \\
\hline & 3 & $\mathrm{O}$ & $\mathrm{O}$ & $\mathrm{O}$ & $\mathrm{O}$ & $\mathrm{O}$ & $\mathrm{O}$ & $\mathrm{O}$ & $\mathrm{O}$ & $\mathrm{O}$ & $\mathrm{O}$ & 1 & 1 & 1 & 3 \\
\hline Total & & 12 & 18 & 13 & 9 & 12 & 12 & 6 & 8 & 4 & 3 & 4 & 3 & 6 & 110 \\
\hline
\end{tabular}

Notes: A + indicates participant HM-IAs that exceed a $95 \%$ critical value determined by a Monte Carlo analysis of 15,000 synthetics with a uniform-random choice rule on only the corners of the probability budgets.

\footnotetext{
7The details of the synthetics' joint sampling distribution of HM-IA are in Appendix A.4.
} 


\section{Discussion and Conclusion}

This paper introduced a homotheticity condition for lottery choices and showed that it is a natural counterpart to the betweenness axiom - betweenness and homotheticity are necessary and sufficient conditions for independence. Decomposing the independence axiom into homotheticity and betweenness allows for a deeper understanding of how independence can be violated. And we exploited this decomposition with an experiment that permitted joint, non-parametric assessments of both independence and homotheticity. These assessments, in some cases, let us infer when the betweenness axiom was violated. Specifically, if choices violated independence but satisfied homotheticity, it was because betweenness was violated.

Figure 8 provides a summary of our empirical results and illustrates how participants can be partitioned into various behavioral classes. Figure 8 also lists parsimonious models that can be used to rationalize choices in each of these behavioral classes. For example, the dark oval in the center of Figure 8 shows that about $1 / 3$ of participants made choices consistent with independence. Expected utility is a parsimonious model that can rationalize choices for these participants. Consider instead the pink chevron in Figure 8. This pink chevron shows that about $1 / 3$ of participants made choices consistent with homotheticity but not independence. Models that can rationalize choices in this behavioral class, roughly speaking, all use some type of probability weighting with a power form.

Our finding that $1 / 3$ of participants made choices consistent with homotheticity, but not independence, presents an apparent point of friction for typical parameterizations of rank-dependent utility (Quiggin, 1982) and cumulative prospect theory (Tversky and Kahneman, 1992). Standard parameterizations of rank-dependent preferences only satisfy homotheticity when the probability weighting function is of the power form (Diecidue, Schmidt, and Zank, 2009). Yet, there is extensive empirical support for an inverse S-shaped probability weighting function (Bruhin, Fehr-Duda, and Epper, 2010). Inverse-S shaped probability weighting implies that on some subdomains of the probability space indifference curves will exhibit quasi-concavity, while on other subdomains indifference curves will exhibit quasi-convexity (see, for example, Figure 2a in FehrDuda and Epper (2012) for the case of three outcomes). Put another way: A standard RDU model 
Figure 8: A summary of behavioral classes and parsimonious models that can be used to rationalize choices therein

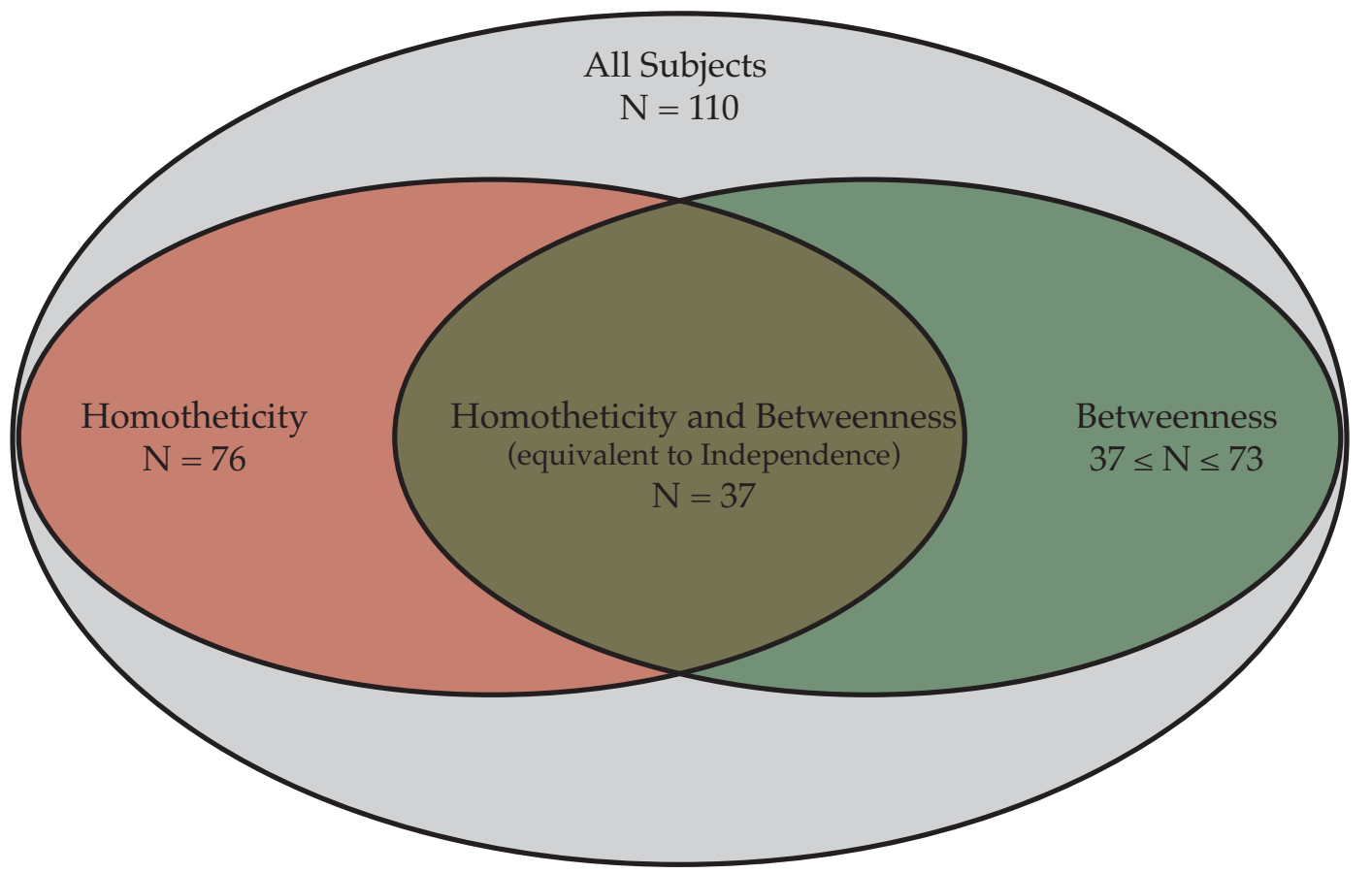

Homotheticity and Betweenness $(\mathrm{N}=37)$

- Expected Utility: von Neumann and Morgenstern (1947)

Homotheticity, not independence. Thus, not betwenness $(\mathrm{N}=39)$

- Rank Dependent Utility with Power Probability Weighting:

Diecidue, et al. (2009), Safra and Segal (1998), Grant and Kajii (1998)

D Betweenness, not independence $(0 \leq \mathrm{N} \leq 36)$

- Disappointment Aversion: Gul (1991)

- Implicit Weighted Utility: Chew and Epstein (1989), Dekel (1986)

Not homotheticity and not betweenness $(0 \leq \mathrm{N} \leq 36)$

- Rank Dependent Models: Abdellaoui (2002),

Tversky and Kahneman (1992), Quiggin (1982)

- Quadratic Utility: Chew et al. (1991)

- Cautious Expected Utility: Cerreia-Vioglio et al. (2013)

- B-L-S Disappointment Aversion: Bell (1985), Loomes and Sugden (1986) 
that is homothetic exhibits either global quasi-concavity or global quasi-convexity (or both, in the case of expected utility). The apparent friction between homotheticity and standard RDU models will, however, need to be explored in a richer empirical setting than the one we considered here. Our empirical results derive from choices over three outcome lotteries. When there are only three outcomes, one cannot falsify a key RDU representational condition of comonotonic independence without also falsifying monotonicity, an assumption we maintain throughout (Wakker, Erev, and Weber, 1994; Chateauneuf, 1999; Abdellaoui, 2002). Diecidue, Schmidt, and Zank (2009) do hint, however, that an RDU model with an inverse S-shaped probability weighting function could be constructed using a 'piece-wise' approach. But, this requires homotheticity to be weakened slightly (see Diecidue, Schmidt, and Zank (2009), p.1112).

More broadly, the class of homothetic, non-independence preferences, represented by the pink chevron in Figure 8, appears to have received little attention in the theoretical risky choice literature. We could find only three papers where these types of preferences have been studied explicitly. The results in this paper indicate that, moving forward, focusing more attention on this class preferences can provide a richer characterization of choices under risk. 


\section{References}

Abdellaoui, M. (2002): "A Genuine Rank-Dependent Generalization of the von NeumannMorgenstern Expected Utility Theorem," Econometrica, 70(2), 717-736.

Allais, M. (1953): “Le Comportement de l’Homme Rationnel devant le Risque: Critique des Postulats et Axiomes de 1'Ecole Americaine," Econometrica, 21(4), 503-546.

_ (1978): “The So-Called Allais Paradox and Rational Decisions Under Uncertainty," pp. 437-681. D. Reidel Publishing Company.

BeLl, D. (1985): “Disappointment in Decision Making under Uncertainty," Operations Research, $33(1), 1-27$.

Bruhin, A., H. Fehr-Duda, and T. Epper (2010): “Risk and Rationality: Uncovering Heterogeneity in Probability Distortion," Econometrica, 78(4), 1375-1412.

Burghart, D. R., P. W. Glimcher, and S. C. Lazzaro (2013): “An Expected Utility Maximizer Walks Into a Bar...," Journal of Risk and Uncertainty, 46(3), 215-246.

Camerer, C., ANd T.-H. Ho (1994): “Violations of the Betweenness Axiom and Nonlinearity in Probability," Journal of Risk and Uncertainty, 8, 167-196.

Cerreia-Vioglio, S., D. Dillenberger, and P. Ortoleva (2013): “Cautious Expected Utility and the Certainty Effect," mimeo, pp. 1-42.

Chateauneuf, A. (1999): “Comonotonicity axioms and rank-dependent expected utility theory for arbitrary consequences," Journal of Mathematical Economics, 32, 21-45.

Chew, S. (1989): "Axiomatic Utility Theories with the Betweenness Property," Annals of Operations Research, 19, 273-298.

Chew, S., L. Epstein, and U. Segal (1991): “Mixture Symmetry and Quadratic Utility," Econometrica. 
Chew, S. H., And L. G. Epstein (1989): “A unifying approach to axiomatic non-expected utility theories," Journal of Economic Theory, 49(2), 207-240.

Chipman, J. (1974): "Homothetic Preferences and Aggregation," Journal of Economic Theory, 8, $26-38$.

Choi, S., R. Fisman, D. Gale, and S. Kariv (2007): “Consistency and Heterogeneity of Individual Behavior Under Uncertainty," American Economic Review, 97(5), 1921-1938.

Dekel, E. (1986): “An Axiomatic Characterization of Preferences Under Uncertainty: Weakening the Independence Axiom," Journal of Economic Theory, 40, 304-318.

Diecidue, E., U. Schmidt, ANd H. Zank (2009): “Parametric Weighting Functions," Journal of Economic Theory, 144, 1102-1118.

Edwards, W. (1962): “Subjective Probabilities Inferred from Decisions," Psychological Review, 69, 109-135.

Fehr-DudA, H., ANd T. EpPer (2012): “Probability and Risk: Foundations and Economic Implications of Probability Weighting," Annual Review of Economics, 4, 567-593.

FishbuRn, P., ANd P. WAKKer (1995): “The Invention of the Independence Condition for Preferences," Management Science, 41(7), 1130-1144.

Gorman, W. M. (1953): “Community Preference Fields,” Econometrica, 21(1), 63-80.

Grant, S., AND A. KaJII (1998): “AUSI expected utility: An anticipated utility theory of relative disappointment aversion," Journal of Economic Behavior and Organization, 37(3), 277-29o.

GuL, F. (1991): “A Theory of Disappointment Aversion," Econometrica, 59(3), 667-686.

Handa, J. (1977): “Risk, Probabilities, and a New Theory of Cardinal Utility," Journal of Political Economy, 85(1), 97-122.

Harbaugh, W. T., K. Krause, and T. Berry (2001): “GARP for Kids: on the Development of Rational Choice Behavior," American Economic Review, 91(5), 1539-1545. 
Heufer, J. (2012): “Testing revealed preferences for homotheticity with two-good experiments," Experimental Economics, 16(1), 114-124.

Hey, J. D., And C. Orme (1994): “Investigating Generalizations of Expected Utility Theory Using Experimental Data," Econometrica, 62(6), 1291-1326.

Houtman, M., ANd J. A. H. MaKs (2007): “Determining all Maximal Data Subsets Consistent with Revealed Preference," Kwantitatieve Methoden, 19, 89-104.

Kahneman, D., And A. Tversky (1979): "Prospect Theory: An Analysis of Decision under Risk," Econometrica, 47(2), 263-292.

Loomes, G., ANd R. Sugden (1986): “Disappointment and Dynamic Consistency in Choice under Uncertainty," Review of Economic Studies, 53(2), 271-282.

Machina, M. (1982): “"Expected Utility" Analysis without the Independence Axiom," Econometrica, 50(2), 277-323.

Manne, A. S. (1952): “The Strong Independence Assumption-Gasoline Blends and Probability Mixtures," Econometrica, 20(4), 665-6.

MarschaK, J. (1950): “Rational Behavior, Uncertain Prospects, and Measurable Utility," Econometrica, 18(2), 111-141.

- (1951): “Why 'Should' Statisticans and Businessmen Maximize 'Moral Expectation'?," in Economic Information, Decision, and Prediction, ed. by J. Neyman.

PolemarchaKis, H. M. (1983): "Homotheticity and the Aggregation of Consumer Demands," Quarterly Journal of Economics, 98(2), 363-369.

Quiggin, J. (1982): “A Theory of Anticipated Utility," Journal of Economic Behavior and Organization, 3, 323-343.

Rubinstein, A., Z. SAfra, and W. Thomson (1992): “On the Interpretation of the Nash Bargaining Solution and Its Extension to Non-Expected Utility Preferences," Econometrica, 6o(5), 1171-1186. 
Safra, Z., and U. Segal (1998): "Constant Risk Aversion," Journal of Economic Theory, 83(1), 19-42.

Samuelson, P. A. (1952): "Probability, Utility, and the Independence Axiom," Econometrica, 20(4), $670-678$.

Starmer, C. (2000): "Developments in Non-Expected Utility Theory: The Hunt for a Descriptive Theory of Choice under Risk," Journal of Economic Literature, 38(2), 332-382.

Sugden, R. (2004): "Alternatives to Expected Utility: Foundations," in Handbook of Utility Theory: Volume II: Extensions, pp. 685-755. Kluwer, Dordrecht.

Tversky, A., and D. Kahneman (1992): “Advances in Prospect Theory: Cumulative Representation of Uncertainty," Journal of Risk and Uncertainty, 5, 297-323.

VARIAN, H. R. (1982): “The Nonparametric Approach to Demand Analysis," Econometrica, 50(4), 945-973.

_ (1983): "Non-Parametric Tests of Consumer Behaviour," Review of Economic Studies, 50(1), 99-110.

von Neumann, J., and O. Morgenstern (1947): “Theory of Games and Economic Behavior," Princeton University Press, Priceton (NJ).

Wakker, P., I. Erev, and E. H. Weber (1994): “Comonotonic Independence: the Critical Test Between Classical and Rank-Dependent Utility Theories," Journal of Risk and Uncertainty.

Wold, H., G. L. S. Shackle, and L. J. Savage (1952): “Ordinal Preferences or Cardinal Utility?," Econometrica, 20(4), 661-664. 


\section{A Appendices for Online Publication}

\section{A.1 Exclusion of participants with unique-corner choices}

We collected data from 162 participants. But, despite our best efforts to design an experiment with the sensitivity to detect our behaviors of interest, some participants made choices that lie outside the dynamic range of our behavioral detector. Patterns of choices consistent with such a phenomenon are those that are all at one "corner" of the 14 probability budgets. ${ }^{8}$ Of the 162 participants, 18 (11\%) exhibit choices which are at a unique corner for all 14 budgets in either a low or high choice-environment. Given the high proportion of subjects (89\%) for whom our behavioral detector does has power, we exclude those 14 participants who exhibited a unique-corner behavior from subsequent analyses and focus on the 144 participants for which our behavioral detector had power. 9

\section{A.2 Exclusion of participants whose choices are inconsistent with direct tests of monotonicity}

Table 3 reports the number of individuals whose choices were consistent with our direct tests of monotonicity (i.e. the horizontal line-segments in Figure 5). As can be seen in Table 3, 129 participants make choices perfectly consistent with monotonicity (i.e. two "correct" choices in the low choice environment and two "correct" choices in the high environment). This represents $90 \%$ of the 144 participants whose choices were not at a unique corner. Because of the importance of monotonicity assumptions in models of risky decision-making we only interrogate the choices made by these 129 participants. ${ }^{10}$

\footnotetext{
${ }^{8}$ It is also quite plausible that participants exhibiting this "unique corner" choice-pattern did not understand the experiment or were rushing through the experiment as quickly as possible.

${ }^{9}$ Note that unique corner choices trivially adhere to GARP, HARP, and independence.

${ }^{10}$ Filtering so strongly on a monotonicity criterion can also be conceived as a filter that removes from analysis those participants who may not have been paying complete attention. Furthermore, an analysis of these 144 participants does not qualitatively alter our findings.
} 
Table 3: Counts of Choices Consistent With Monotonicity

\begin{tabular}{cc||ccc|c}
\multicolumn{1}{c|}{ Number of Choices Consistent With Monotonicity } \\
\hline & & \multicolumn{2}{c}{ Low } & & Total \\
& & 2 & 1 & 0 & \\
\hline \hline \multirow{3}{*}{ High } & 2 & 129 & 2 & 0 & 131 \\
& 1 & 9 & 3 & 0 & 12 \\
& o & 1 & 0 & 0 & 1 \\
\hline Total & & 139 & 5 & 0 & 144 \\
\hline
\end{tabular}

\section{A.3 Exclusion of participants whose choices are inconsistent with the generalized axiom of revealed preference (GARP)}

In the body of the paper we focus on those participants whose choices can be considered to be consistent with the generalized axiom of revealed preference (GARP). As Afriat's theorem guarantees, these participant's choices can be modeled with a preference relation that is complete, transitive, continuous, monotone, and quasi-concave.

For the 129 participants whose choices were consistent with our direct tests of monotonicity, $87(76 \%)$ made choices that were perfectly consistent with GARP in both the low and high choiceenvironments. If the threshold of GARP compliance is relaxed to a commonly invoked $95 \%$ critical value, relative to a distribution of 15,000 synthetics with an independent and uniform random choice rule, then 110 participants $(85 \%)$ made choices consistent with GARP. ${ }^{11}$ We focus on those 110 participants in the body of the paper. ${ }^{12}$

Varian (1982) outlines an algorithm for identifying violations of GARP and we generally follow his notation and approach here. ${ }^{13}$ For the 14 budgets with "normal" prices in our experiment, $\left(q^{1}, \ldots, q^{14}\right)$ and each participants choices $\left(x^{1}, \ldots, x^{14}\right)$, we construct a 14 by 14 matrix $M$ (the directly revealed graph) whose $i j^{\text {th }}$ entry is given by

\footnotetext{
${ }^{11}$ This Monte Carlo analysis reveals that one "mistake" is acceptable across both the low and high choice environments which permits Houtman-Maks indices of $\{14,14\},\{14,13\}$, and $\{13,14\}$.

${ }^{12}$ Including participants whose choices are inconsistent with GARP would make little sense. Choices consistent with the homothetic axiom of revealed preference can be modeled with a preference relation that is complete, transitive, continuous, monotone, quasi-concave, and homothetic. These conditions are more restrictive than those guaranteed by GARP.

13 However, we do depart slightly from Varian's approach: Instead of employing Warshall's algorithm for calculating the transitive closure of a graph we use the computationally more intensive (but easier to program) method of inferring the transitive closure by multiplying the directly revealed preferred graph by itself 14 times.
} 


$$
m_{i j}= \begin{cases}1 & \text { if } q^{i} \cdot x^{i} \geq q^{i} \cdot x^{j} \\ 0 & \text { otherwise }\end{cases}
$$

We then construct the indirectly revealed preferred graph $M T$, which is the transitive closure of $M$. The $i j^{\text {th }}$ entry of the closure is given by

$$
m t_{i j}= \begin{cases}1 & \text { if } m_{i j}^{14}>0 \\ 0 & \text { otherwise }\end{cases}
$$

where $m_{i j}^{14}$ is the $i j^{\text {th }}$ entry of the matrix $M^{14}=M M \cdots M$. If $m t_{i j}=1$ and $q^{j} \cdot x^{j}>q^{j} \cdot x^{i}$ for some $i$ and $j$ (with $x^{i} \neq x^{j}$ ) there is a GARP violation.

A commonly deployed analytical approach in analyses of axiomatic violations is to determine "how badly" choices depart from perfect rationality. And a common measure used in this regard is the Houtman-Maks (HM) index. Houtman-Maks is the largest subset of choices which are GARP-compliant. So, for example, if we see that a full set of 14 choices do not conform to GARP but, by removing one offending choice the remaining are consistent with GARP, this would represent a Houtman-Maks of 13.

To calculate Houtman-Maks, conditional on observing at least one violation of GARP, we take a brute force approach: We check whether all subsets of size 13 are consistent with GARP (using the above algorithm). If no subset of size 13 is GARP compliant we take all subsets of size 12 and check whether any of these subsets are GARP compliant. We proceed in such a manner until we find at least one subset of the data that is GARP compliant. The cardinality of that subset is the Houtman-Maks.

To determine a sampling distribution for Houtman-Maks indices we compare our participant HMs to a Monte Carlo analysis. Our Monte Carlo analysis simulates choices for 15,000 synthetic participants that have a uniform-random choice rule. For each of these synthetic's choices we calculate Houtman-Maks. ${ }^{14}$ Based on this joint sampling distribution, the details of which are

${ }^{14}$ Note that for the synthetics the choice rule is independent across trials. Thus, the joint sampling distribution across two choice environments is the multiplication of the marginal distribution resulting from from the analysis of synthetics choices in one choice environment. 
in Appendix A.4, a 95\% critical value permits three patterns of Houtman-Maks indices for our human choosers: $\{14,14\},\{14,13\}$, and $\{13,14\}$. Table 4 shows the number of participants which have specific Houtman-Maks values in the low and high choice environments and those patterns which meet or exceed the $95 \%$ threshold are indicated with a dagger $(\dagger) .{ }^{15}$ For the 129 participant choices analyzed for compliance with GARP, $110(85 \%)$ have a Houtman-Maks that meets or exceeds the thresholds determined by the $95 \%$ critical value.

Table 4: Counts of individuals at Houtman-Maks (HM) index values

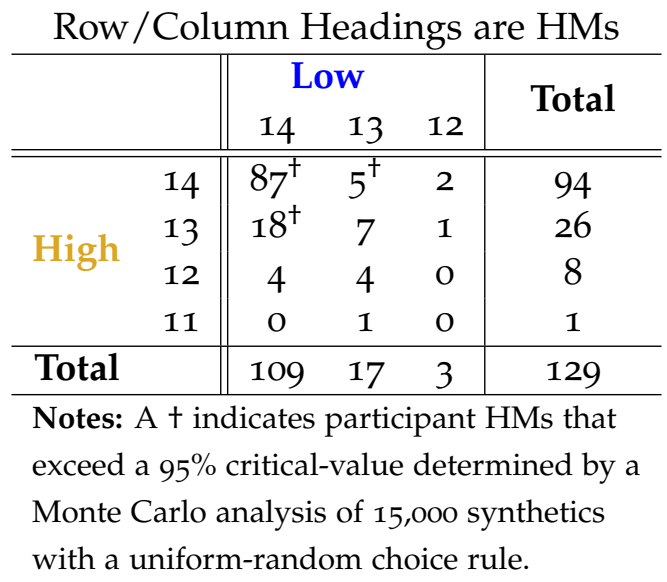

\footnotetext{
15 As detailed in Table 5 in Appendix A.4 the precise critical value for these choice patterns is 92.7\%. However, for expositional expediency we refer to this as the commonly invoked $95 \%$ criterion.
} 


\section{A.4 Sampling distributions from Monte Carlo analyses}

Table 5: Joint-distribution of Houtman-Maks (GARP) indices for 15,000 synthetic choosers with a uniform-random choice rule

\begin{tabular}{cc||ccccccc|c}
\multicolumn{10}{c}{ Row and column headings are HM Indices } \\
\hline & & 14 & 13 & 12 & 11 & 10 & 9 & 8 & Total \\
\hline \hline \multirow{6}{*}{ High } & 14 & 1.03 & 3.14 & 3.50 & 1.95 & 0.49 & 0.05 & $<0.01$ & 10.17 \\
& 13 & 3.14 & 9.52 & 10.63 & 5.92 & 1.48 & 0.16 & 0.01 & 30.86 \\
& 12 & 3.50 & 10.63 & 11.87 & 6.61 & 1.65 & 0.18 & 0.01 & 34.45 \\
& 11 & 1.95 & 5.92 & 6.61 & 3.68 & 0.92 & 0.10 & $<0.01$ & 19.19 \\
& 10 & 0.49 & 1.48 & 1.65 & 0.92 & 0.23 & 0.03 & $<0.01$ & 4.78 \\
& 9 & 0.05 & 0.16 & 0.18 & 0.10 & 0.03 & $<0.01$ & $<0.01$ & 0.52 \\
& 8 & $<0.01$ & 0.01 & 0.01 & $<0.01$ & $<0.01$ & $<0.01$ & $<0.00$ & 0.02 \\
\hline Total & 10.17 & 30.86 & 34.45 & 19.19 & 4.78 & 0.52 & 0.02 & 100.00 \\
\hline
\end{tabular}

Notes: Cell entries are percentages (\%) 
Table 6: Joint-distribution of Houtman-Maks adapted to HARP for 15,000 synthetic choosers with a uniform random choice rule Row and column headings are HM-HARP

\begin{tabular}{|c|c|c|c|c|c|c|c|c|c|c|c|c|c|}
\hline & & \multicolumn{11}{|c|}{ Low } & \multirow[b]{2}{*}{ Total } \\
\hline & & 14 & 13 & 12 & 11 & 10 & 9 & 8 & 7 & 6 & 5 & 4 & \\
\hline \multirow{11}{*}{ High } & 14 & 0.00 & 0.00 & 0.00 & 0.00 & 0.00 & 0.00 & 0.00 & 0.00 & 0.00 & 0.00 & 0.00 & 0.00 \\
\hline & 13 & 0.00 & 0.00 & 0.00 & 0.00 & 0.00 & 0.00 & 0.00 & 0.00 & 0.00 & 0.00 & 0.00 & 0.00 \\
\hline & 12 & 0.00 & 0.00 & $<0.01$ & $<0.01$ & $<0.01$ & $<0.01$ & $<0.01$ & 0.01 & 0.01 & $<0.01$ & $<0.01$ & 0.02 \\
\hline & 11 & 0.00 & 0.00 & $<0.01$ & $<0.01$ & $<0.01$ & 0.01 & 0.03 & 0.06 & 0.05 & 0.02 & $<0.01$ & 0.19 \\
\hline & 10 & 0.00 & 0.00 & $<0.01$ & $<0.01$ & 0.03 & 0.10 & 0.30 & 0.53 & 0.48 & 0.17 & 0.01 & 1.62 \\
\hline & 9 & 0.00 & 0.00 & $<0.01$ & 0.01 & 0.10 & 0.41 & 1.19 & 2.11 & 1.88 & 0.65 & 0.04 & 6.40 \\
\hline & 8 & 0.00 & 0.00 & $<0.01$ & 0.03 & 0.30 & 1.19 & 3.46 & 6.13 & 5.46 & 1.90 & 0.12 & 18.61 \\
\hline & 7 & 0.00 & 0.00 & 0.01 & 0.06 & 0.53 & 2.11 & 6.13 & 10.85 & 9.67 & $3 \cdot 36$ & 0.22 & 32.93 \\
\hline & 6 & 0.00 & 0.00 & 0.01 & 0.05 & 0.48 & 1.88 & 5.46 & 9.67 & 8.61 & 3.00 & 0.20 & $29 \cdot 35$ \\
\hline & 5 & 0.00 & 0.00 & $<0.01$ & 0.02 & 0.17 & 0.65 & 1.90 & $3 \cdot 36$ & 3.00 & 1.04 & 0.07 & 10.21 \\
\hline & 4 & 0.00 & 0.00 & $<0.01$ & $<0.01$ & 0.01 & 0.04 & 0.12 & 0.22 & 0.20 & 0.07 & $<0.01$ & 0.66 \\
\hline Total & & 0.00 & 0.00 & 0.02 & 0.19 & 1.62 & 6.40 & 18.61 & 32.93 & 29.35 & 10.21 & 0.66 & 100.00 \\
\hline
\end{tabular}

Notes: Cell entries are percentages (\%). 
Table 7: Joint-distribution of Houtman-Maks adapted to our test of independence for 15,000 synthetic choosers with a random choice rule for only the corner alternatives

Row and column headings are HM-IA

\begin{tabular}{|c|c|c|c|c|c|c|c|c|c|c|}
\hline & & \multicolumn{8}{|c|}{ Low } & \multirow[b]{2}{*}{ Total } \\
\hline & & 14 & 13 & 12 & 11 & 10 & 9 & 8 & 7 & \\
\hline \multirow{8}{*}{ High } & 14 & $<0.01$ & $<0.01$ & 0.01 & 0.02 & 0.03 & 0.03 & 0.02 & $<0.01$ & 0.12 \\
\hline & 13 & $<0.01$ & 0.02 & 0.09 & 0.24 & 0.36 & 0.36 & 0.18 & 0.01 & 1.25 \\
\hline & 12 & 0.01 & 0.09 & 0.47 & 1.29 & 1.97 & 1.96 & 0.98 & 0.08 & 6.84 \\
\hline & 11 & 0.02 & 0.24 & 1.29 & $3 \cdot 58$ & $5 \cdot 45$ & $5 \cdot 41$ & 2.71 & 0.22 & 18.92 \\
\hline & 10 & 0.03 & 0.36 & 1.97 & $5 \cdot 45$ & 8.29 & 8.24 & 4.13 & 0.33 & 28.80 \\
\hline & 9 & 0.03 & 0.36 & 1.96 & $5 \cdot 41$ & 8.24 & 8.18 & 4.10 & 0.33 & 28.60 \\
\hline & 8 & 0.02 & 0.18 & 0.98 & 2.71 & 4.13 & 4.10 & 2.05 & 0.16 & 14.33 \\
\hline & 7 & $<0.01$ & 0.01 & 0.08 & 0.22 & 0.33 & 0.33 & 0.16 & 0.01 & 1.14 \\
\hline Total & & 0.12 & 1.25 & 6.84 & 18.92 & 28.80 & 28.60 & 14.33 & 1.14 & 100.00 \\
\hline
\end{tabular}

Notes: Cell entries are percentages (\%). 


\section{A.5 Experimental Design Details}

Table 8: Probability budget details

\begin{tabular}{|c|c|c|c|c|c|c|}
\hline \multirow{2}{*}{$\begin{array}{c}\text { CS } \\
\text { Number }\end{array}$} & \multirow{2}{*}{$\begin{array}{c}\text { Choice- } \\
\text { Environment }\end{array}$} & \multicolumn{3}{|c|}{ Outcomes (CHF) } & \multicolumn{2}{|c|}{ Prices } \\
\hline & & $z_{1}$ & $z_{2}$ & $z_{3}$ & $q_{1}$ & $q_{2}$ \\
\hline 1 & low & 40 & 20 & $\mathrm{o}$ & 0.7 & 0.2 \\
\hline 2 & low & 40 & 20 & $\mathrm{O}$ & 0.7 & 0.1 \\
\hline 3 & low & 40 & 20 & $\mathrm{O}$ & 0.6 & 0.4 \\
\hline 4 & low & 40 & 20 & $\mathrm{o}$ & 0.6 & 0.3 \\
\hline 5 & low & 40 & 20 & $\mathrm{o}$ & 0.6 & 0.2 \\
\hline 6 & low & 40 & 20 & $\mathrm{o}$ & 0.6 & 0.1 \\
\hline 7 & low & 40 & 20 & $\mathrm{o}$ & 0.5 & 0.5 \\
\hline 8 & low & 40 & 20 & $\mathrm{o}$ & 0.5 & 0.4 \\
\hline 9 & low & 40 & 20 & $\mathrm{O}$ & 0.5 & 0.3 \\
\hline 10 & low & 40 & 20 & $\mathrm{O}$ & 0.5 & 0.2 \\
\hline 11 & low & 40 & 20 & $\mathrm{o}$ & 0.5 & 0.1 \\
\hline 12 & low & 40 & 20 & $\mathrm{o}$ & 0.4 & 0.4 \\
\hline 13 & low & 40 & 20 & $\mathrm{O}$ & 0.4 & 0.3 \\
\hline 14 & low & 40 & 20 & $\mathrm{o}$ & 0.4 & 0.2 \\
\hline 15 & low & 40 & 20 & $\mathrm{o}$ & - & - \\
\hline 16 & low & 40 & 20 & $\mathrm{o}$ & - & - \\
\hline 17 & high & 60 & 40 & 20 & 0.7 & 0.2 \\
\hline 18 & high & 60 & 40 & 20 & 0.7 & 0.1 \\
\hline 19 & high & 60 & 40 & 20 & 0.6 & 0.4 \\
\hline 20 & high & 60 & 40 & 20 & 0.6 & 0.3 \\
\hline 21 & high & 60 & 40 & 20 & 0.6 & 0.2 \\
\hline 22 & high & 60 & 40 & 20 & 0.6 & 0.1 \\
\hline 23 & high & 60 & 40 & 20 & 0.5 & 0.5 \\
\hline 24 & high & 60 & 40 & 20 & 0.5 & 0.4 \\
\hline 25 & high & 60 & 40 & 20 & 0.5 & 0.3 \\
\hline 26 & high & 60 & 40 & 20 & 0.5 & 0.2 \\
\hline 27 & high & 60 & 40 & 20 & 0.5 & 0.1 \\
\hline 28 & high & 60 & 40 & 20 & 0.4 & 0.4 \\
\hline 29 & high & 60 & 40 & 20 & 0.4 & 0.3 \\
\hline 30 & high & 60 & 40 & 20 & 0.4 & 0.2 \\
\hline 31 & high & 60 & 40 & 20 & - & - \\
\hline 32 & high & 60 & 40 & 20 & - & - \\
\hline
\end{tabular}


Table 9: Choice-situation construction details

\begin{tabular}{|c|c|c|c|c|}
\hline $\begin{array}{c}\text { CS } \\
\text { Number }\end{array}$ & $\begin{array}{c}\text { Choice- } \\
\text { Environment }\end{array}$ & $\begin{array}{c}\text { Outcomes (CHF) } \\
\left\{z_{1}, z_{2}, z_{3}\right\}\end{array}$ & $\begin{array}{c}L^{10} \\
\left(\operatorname{Prob}_{1}, \operatorname{Prob}_{2}, \text { Prob }_{3}\right) \\
\end{array}$ & $\begin{array}{c}L^{0} \\
\left(\mathrm{Prob}_{1}, \mathrm{Prob}_{2}, \mathrm{Prob}_{3}\right) \\
\end{array}$ \\
\hline 1 & low & $\{40,20,0\}$ & $(0.0,0.9,0.1)$ & $(0.7,0.0,0.3)$ \\
\hline 2 & low & $\{40,20,0\}$ & $(0.0,0.8,0.2)$ & $(0.7,0.0,0.3)$ \\
\hline 3 & low & $\{40,20,0\}$ & $(0.0,1.0,0.0)$ & $(0.6,0.0,0.4)$ \\
\hline 4 & low & $\{40,20,0\}$ & $(0.0,0.9,0.1)$ & $(0.6,0.0,0.4)$ \\
\hline 5 & low & $\{40,20,0\}$ & $(0.0,0.8,0.2)$ & $(0.6,0.0,0.4)$ \\
\hline 6 & low & $\{40,20,0\}$ & $(0.0,0.7,0.3)$ & $(0.6,0.0,0.4)$ \\
\hline 7 & low & $\{40,20,0\}$ & $(0.0,1.0,0.0)$ & $(0.5,0.0,0.5)$ \\
\hline 8 & low & $\{40,20,0\}$ & $(0.0,0.9,0.1)$ & $(0.5,0.0,0.5)$ \\
\hline 9 & low & $\{40,20,0\}$ & $(0.0,0.8,0.2)$ & $(0.5,0.0,0.5)$ \\
\hline 10 & low & $\{40,20,0\}$ & $(0.0,0.7,0.3)$ & $(0.5,0.0,0.5)$ \\
\hline 11 & low & $\{40,20,0\}$ & $(0.0,0.6,0.4)$ & $(0.5,0.0,0.5)$ \\
\hline 12 & low & $\{40,20,0\}$ & $(0.0,0.8,0.2)$ & $(0.4,0.0,0.6)$ \\
\hline 13 & low & $\{40,20,0\}$ & $(0.0,0.7,0.3)$ & $(0.4,0.0,0.6)$ \\
\hline 14 & low & $\{40,20,0\}$ & $(0.0,0.6,0.4)$ & $(0.4,0.0,0.6)$ \\
\hline 15 & low & $\{40,20,0\}$ & $(0.7,0.3,0.0)$ & $(0.7,0.0,0.3)$ \\
\hline 16 & low & $\{40,20,0\}$ & $(0.5,0.5,0.0)$ & $(0.5,0.0,0.5)$ \\
\hline 17 & high & $\{60,40,20\}$ & $(0.0,0.9,0.1)$ & $(0.7,0.0,0.3)$ \\
\hline 18 & high & $\{60,40,20\}$ & $(0.0,0.8,0.2)$ & $(0.7,0.0,0.3)$ \\
\hline 19 & high & $\{60,40,20\}$ & $(0.0,1.0,0.0)$ & $(0.6,0.0,0.4)$ \\
\hline 20 & high & $\{60,40,20\}$ & $(0.0,0.9,0.1)$ & $(0.6,0.0,0.4)$ \\
\hline 21 & high & $\{60,40,20\}$ & $(0.0,0.8,0.2)$ & $(0.6,0.0,0.4)$ \\
\hline 22 & high & $\{60,40,20\}$ & $(0.0,0.7,0.3)$ & $(0.6,0.0,0.4)$ \\
\hline 23 & high & $\{60,40,20\}$ & $(0.0,1.0,0.0)$ & $(0.5,0.0,0.5)$ \\
\hline 24 & high & $\{60,40,20\}$ & $(0.0,0.9,0.1)$ & $(0.5,0.0,0.5)$ \\
\hline 25 & high & $\{60,40,20\}$ & $(0.0,0.8,0.2)$ & $(0.5,0.0,0.5)$ \\
\hline 26 & high & $\{60,40,20\}$ & $(0.0,0.7,0.3)$ & $(0.5,0.0,0.5)$ \\
\hline 27 & high & $\{60,40,20\}$ & $(0.0,0.6,0.4)$ & $(0.5,0.0,0.5)$ \\
\hline 28 & high & $\{60,40,20\}$ & $(0.0,0.8,0.2)$ & $(0.4,0.0,0.6)$ \\
\hline 29 & high & $\{60,40,20\}$ & $(0.0,0.7,0.3)$ & $(0.4,0.0,0.6)$ \\
\hline 30 & high & $\{60,40,20\}$ & $(0.0,0.6,0.4)$ & $(0.4,0.0,0.6)$ \\
\hline 31 & high & $\{60,40,20\}$ & $(0.7,0.3,0.0)$ & $(0.7,0.0,0.3)$ \\
\hline 32 & high & $\{60,40,20\}$ & $(0.5,0.5,0.0)$ & $(0.5,0.0,0.5)$ \\
\hline
\end{tabular}

Notes: The lotteries listed above were not alternatives available in the experiment. However, each of the nine alternatives available in a given choice-situation $\left(\left\{L^{9}, \ldots, L^{1}\right\}\right)$ can be constructed as a convex combination of $L^{10}$ and $L^{0}: L^{j}=\alpha_{j} L^{10}+\left(1-\alpha_{j}\right) L^{0}$ where $\alpha_{j} \in\{0.9,0.8, \ldots, 0.1\}$. 
Table 10: A list of the nine lotteries available in each of the choice-situations using the low choice-environment

CS Outcomes List of Lotteries $\left\{L^{9}, L^{8}, \ldots, L^{1}\right\}$

$\{(0.07,0.81,0.12),(0.14,0.72,0.14),(0.21,0.63,0.16),(0.28,0.54,0.18),(0.35,0.45,0.2),(0.42,0.36,0.22),(0.49,0.27,0.24),(0.56,0.18,0.26),(0.63,0.09,0.28)\}$ $\{40,20,0\} \quad\{(0.07,0.72,0.21),(0.14,0.64,0.22),(0.21,0.56,0.23),(0.28,0.48,0.24),(0.35,0.4,0.25),(0.42,0.32,0.26),(0.49,0.24,0.27),(0.56,0.16,0.28),(0.63,0.08,0.29)\}$ $\{40,20,0\} \quad\{(0.06,0.9,0.04),(0.12,0.8,0.08),(0.18,0.7,0.12),(0.24,0.6,0.16),(0.3,0.5,0.2),(0.36,0.4,0.24),(0.42,0.3,0.28),(0.48,0.2,0.32),(0.54,0.1,0.36)\}$ $\{40,20,0\} \quad\{(0.06,0.81,0.13),(0.12,0.72,0.16),(0.18,0.63,0.19),(0.24,0.54,0.22),(0.3,0.45,0.25),(0.36,0.36,0.28),(0.42,0.27,0.31),(0.48,0.18,0.34),(0.54,0.09,0.37)\}$ $\{40,20,0\} \quad\{(0.06,0.72,0.22),(0.12,0.64,0.24),(0.18,0.56,0.26),(0.24,0.48,0.28),(0.3,0.4,0.3),(0.36,0.32,0.32),(0.42,0.24,0.34),(0.48,0.16,0.36),(0.54,0.08,0.38)\}$ $\{40,20,0\} \quad\{(0.06,0.63,0.31),(0.12,0.56,0.32),(0.18,0.49,0.33),(0.24,0.42,0.34),(0.3,0.35,0.35),(0.36,0.28,0.36),(0.42,0.21,0.37),(0.48,0.14,0.38),(0.54,0.07,0.39)\}$

$7 \quad\{40,20,0\} \quad\{(0.05,0.9,0.05),(0.1,0.8,0.1),(0.15,0.7,0.15),(0.2,0.6,0.2),(0.25,0.5,0.25),(0.3,0.4,0.3),(0.35,0.3,0.35),(0.4,0.2,0.4),(0.45,0.1,0.45)\}$

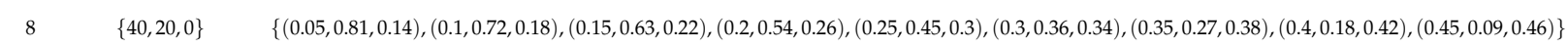

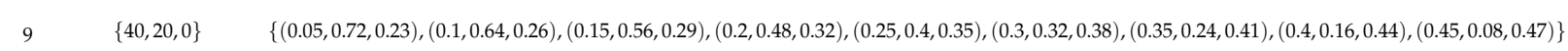

$10 \quad\{40,20,0\} \quad\{(0.05,0.63,0.32),(0.1,0.56,0.34),(0.15,0.49,0.36),(0.2,0.42,0.38),(0.25,0.35,0.4),(0.3,0.28,0.42),(0.35,0.21,0.44),(0.4,0.14,0.46),(0.45,0.07,0.48)\}$

$11 \quad\{40,20,0\} \quad\{(0.05,0.54,0.41),(0.1,0.48,0.42),(0.15,0.42,0.43),(0.2,0.36,0.44),(0.25,0.3,0.45),(0.3,0.24,0.46),(0.35,0.18,0.47),(0.4,0.12,0.48),(0.45,0.06,0.49)\}$

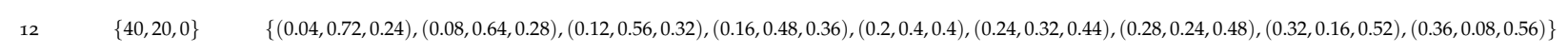

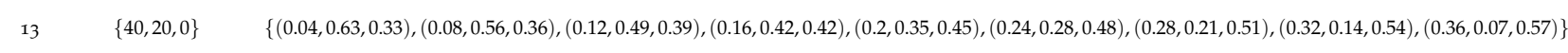

$14\{40,20,0\} \quad\{(0.04,0.54,0.42),(0.08,0.48,0.44),(0.12,0.42,0.46),(0.16,0.36,0.48),(0.2,0.3,0.5),(0.24,0.24,0.52),(0.28,0.18,0.54),(0.32,0.12,0.56),(0.36,0.06,0.58)\}$

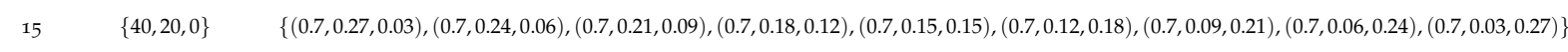

$16 \quad\{40,20,0\} \quad\{(0.5,0.45,0.05),(0.5,0.4,0.1),(0.5,0.35,0.15),(0.5,0.3,0.2),(0.5,0.25,0.25),(0.5,0.2,0.3),(0.5,0.15,0.35),(0.5,0.1,0.4),(0.5,0.05,0.45)\}$ 
Table 11: A list of the nine lotteries available in each of the choice-situations using the high choice-environment

\section{CS Outcomes}

List of Lotteries $\left\{L^{9}, L^{8}, \ldots, L^{1}\right\}$

$\{60,40,20\} \quad\{(0.07,0.81,0.12),(0.14,0.72,0.14),(0.21,0.63,0.16),(0.28,0.54,0.18),(0.35,0.45,0.2),(0.42,0.36,0.22),(0.49,0.27,0.24),(0.56,0.18,0.26),(0.63,0.09,0.28)\}$ $\{60,40,20\} \quad\{(0.07,0.72,0.21),(0.14,0.64,0.22),(0.21,0.56,0.23),(0.28,0.48,0.24),(0.35,0.4,0.25),(0.42,0.32,0.26),(0.49,0.24,0.27),(0.56,0.16,0.28),(0.63,0.08,0.29)\}$ $\{60,40,20\} \quad\{(0.06,0.9,0.04),(0.12,0.8,0.08),(0.18,0.7,0.12),(0.24,0.6,0.16),(0.3,0.5,0.2),(0.36,0.4,0.24),(0.42,0.3,0.28),(0.48,0.2,0.32),(0.54,0.1,0.36)\}$

$\{60,40,20\} \quad\{(0.06,0.81,0.13),(0.12,0.72,0.16),(0.18,0.63,0.19),(0.24,0.54,0.22),(0.3,0.45,0.25),(0.36,0.36,0.28),(0.42,0.27,0.31),(0.48,0.18,0.34),(0.54,0.09,0.37)\}$ $\{60,40,20\} \quad\{(0.06,0.72,0.22),(0.12,0.64,0.24),(0.18,0.56,0.26),(0.24,0.48,0.28),(0.3,0.4,0.3),(0.36,0.32,0.32),(0.42,0.24,0.34),(0.48,0.16,0.36),(0.54,0.08,0.38)\}$ $\{60,40,20\} \quad\{(0.06,0.63,0.31),(0.12,0.56,0.32),(0.18,0.49,0.33),(0.24,0.42,0.34),(0.3,0.35,0.35),(0.36,0.28,0.36),(0.42,0.21,0.37),(0.48,0.14,0.38),(0.54,0.07,0.39)\}$

$23 \quad\{60,40,20\} \quad\{(0.05,0.9,0.05),(0.1,0.8,0.1),(0.15,0.7,0.15),(0.2,0.6,0.2),(0.25,0.5,0.25),(0.3,0.4,0.3),(0.35,0.3,0.35),(0.4,0.2,0.4),(0.45,0.1,0.45)\}$

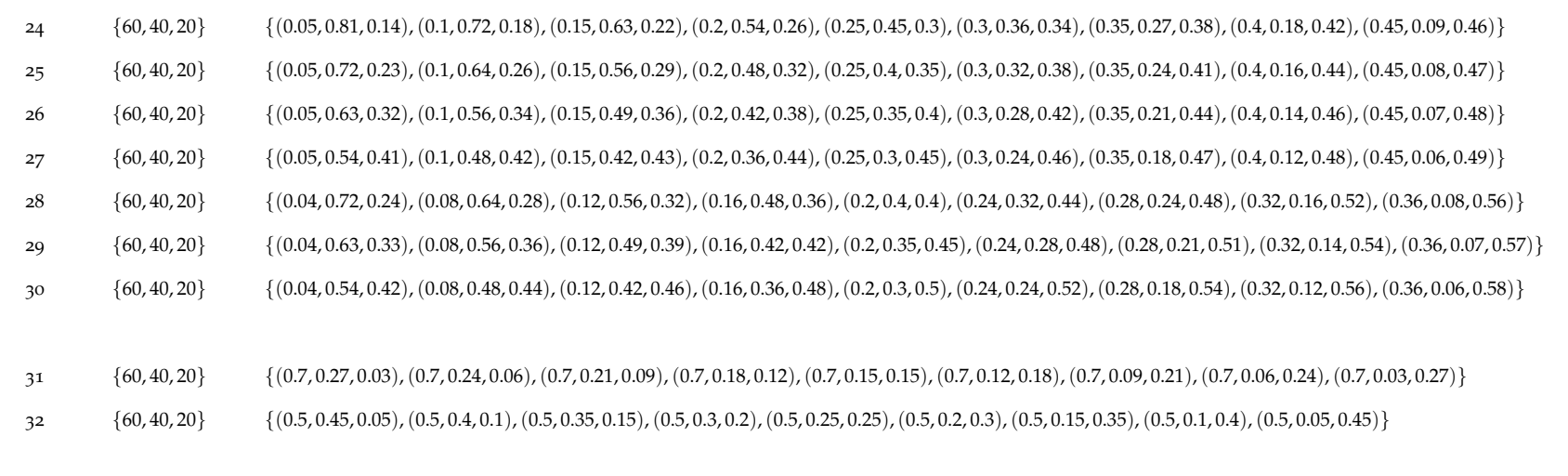




\section{Welcome}

Please wait to open this packet until you are instructed to do so.

Figure 9: Instructions Page 1 


\section{Introduction}

This is an experiment about decision-making. It should take about 60 minutes. On your desk is a card with a number on it. You will be identified only by this number not by your name. We have a list of the names of people who sign up for the experiment. But, this is for scheduling purposes only and we cannot link your name to your code number. Thus, all your decisions today will be completely anonymous. In addition, all of your payment will be given to you privately, today, in cash, here at the lab.

During the experiment you will be presented with a series of choice-situations. In each choicesituation there is a list of alternatives from which you can choose. These alternatives are a list of possible monetary amounts and the probability of getting each of those amounts. All of the alternatives in the experiment will have three possible amounts. We will explain the alternatives in more detail shortly.

When the experiment is over, the computer will randomly pick one choice-situation to count for actual payment. You will receive the alternative you selected in that choice-situation and you will roll the equivalent of a 100-sided die to determine which of the three monetary amounts you will get from that alternative. In addition to the amount you get from your alternative, you will receive CHF 10 as a show-up fee. I will explain payments in more detail shortly.

Please wait to turn the page until you are instructed to do so.

Figure 10: Instructions Page 2 


\section{Alternatives}

In the experiment you will be making choices about alternatives. An alternative is a list of monetary amounts and the probability of getting each amount. All the alternatives in today's experiment will have three possible monetary amounts and these amounts will always be Swiss Francs (CHF). An example of how an alternative will be presented in the experiment is in the figure to the right. This alternative has CHF 0, CHF 20, and $\mathrm{CHF} 40$ as possible amounts. You can receive only one of these three amounts from this alternative. The probability of receiving a given amount is shown as a percentage (out of $100 \%$ ) just to the right of the amount information.

The probability you get CHF 0 from this alternative is $40 \%$, or 40 out of 100 . The probability you get CHF 20 from this alternative is $40 \%$, or 40 out of 100 . And the probability you get CHF 40 from this alternative is $20 \%$, or 20 out of 100 . Note that all of the probabilities add up to $100 \%$. So, there are no hidden amounts. All the possible monetary amounts are right there.

The height of each of the colored blocks is proportional to the probabilities. You can see this in the alternative to the right: The block for the CHF 0 amount is the same height as the block for the CHF 20 amount. This is because the probability of receiving CHF 0 or CHF 20 from this alternative are each $40 \%$. The block for the CHF 40 amount is half the height of the CHF 0 and 20 blocks because the probability of receiving CHF 40 from this alternative is $20 \%$ (i.e. half of $40 \%$ ).

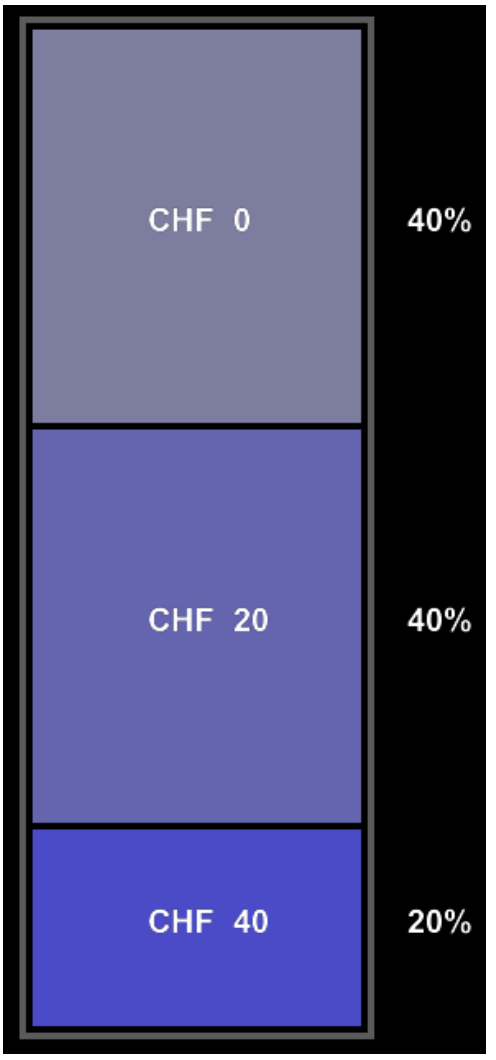

In addition, each of the blocks is a specific color that is associated only with that amount.

Alternatives will always be presented so that the smallest amount is on the top and the largest amount is on the bottom.

Please wait to turn the page until you are instructed to do so.

Figure 11: Instructions Page 3 


\section{Types of Alternatives}

In the experiment there will be two types of alternatives. The first type of alternative will have CHF 0, CHF 20, and CHF 40 as the three possible amounts. This 0-20-40 type of alternative will always be presented with the blue color scheme you see here to the right.

The second type of alternative will have CHF 20, CHF 40, and CHF 60 as the three possible amounts. This 20-40-60 type of alternative will always be presented with the yellow color scheme you see here below to the left.

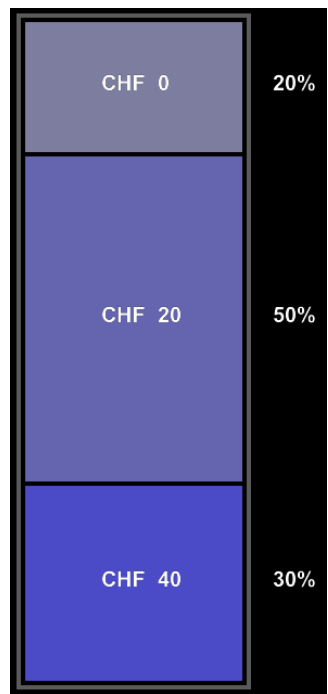

We use these different color schemes to make sure that it is clear to you which amounts are possible from a specific alternative.

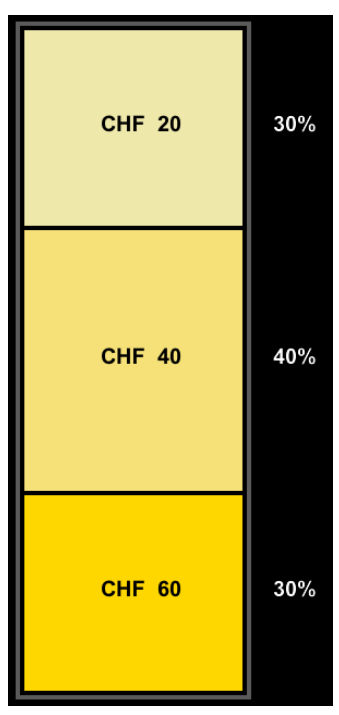

In the experiment you will be presented with choice-situations in which you will select amongst blue-types (0-20-40) of alternatives or amongst yellow-types (2040-60) of alternatives. However you will never be presented with a choice-situation in which you will select amongst both blue-type and yellow-type alternatives together: All of the alternatives in a given choice-situation will either be all blue or all yellow. However, to make these instructions more efficient we will only use the blue-type (0-20-40) alternatives to describe how the experiment works.

Please wait to turn the page until you are instructed to do so.

Figure 12: Instructions Page 4 


\section{The Graphical Interface}

In the experiment you will be presented with a series of choice-situations. In each of these choicesituations there will be a list of nine alternatives. The nine alternatives in each choice-situation will either be all blue-type (0-20-40) or all yellow-type (20-40-60). So, in every choice-situation, each of the nine alternatives will have the same possible amounts. However, the probabilities of receiving those amounts will be different across the nine alternatives in a specific choice-situation.

In each choice-situation your job is to select which one of the nine alternatives you want most. To make your job easier the information in each of the choice-situations will be presented with a graphical interface. This section explains how the graphical interface displays all of the information in each choice-situation.

The Graphical Interface has two principle components:

1. Selection Bar: This shows thumbnail images of all the alternatives in the choice-situation.

2. Detailed Alternative: This shows a zoomed view of the alternative highlighted in the Selection Bar.

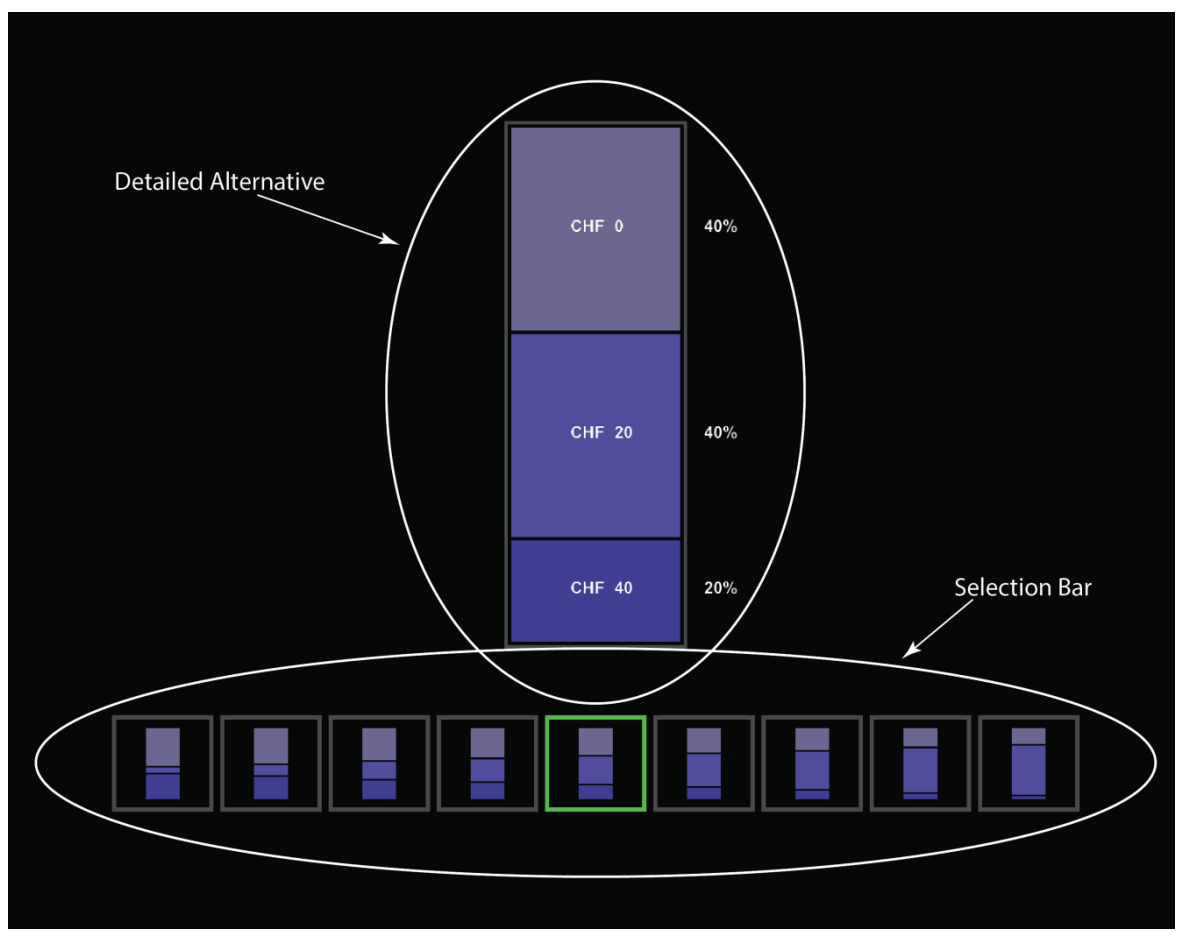

Figure 13: Instructions Page 5 


\section{Detailed Alternative}

At the center of the graphical interface is a large, Detailed Alternative that shows all of the amount and probability information, in text, on top of the colored blocks, just like the alternatives detailed above.

\section{Selection Bar}

At the bottom of each screen is a row of nine boxes. In the center of each of those boxes is a small image of an alternative that the box represents. Note that these thumbnail images do not have probability or amount text displayed on them. To see the precise probability and amount information for any of the nine alternatives just move your mouse over the box with the small image of the alternative on it. When you move your mouse over one of the boxes a green outline will surround the box and the thumbnail alternative will be depicted as the Detailed Alternative so that you can see the exact amount and probability information, in text, on top of the colored boxes.

\section{Making a Choice}

To indicate which alternative you want most from the nine available move your mouse so that the green outline surrounds the box with the small image of your most preferred alternative, so that it is represented as the Detailed Alternative. Then click your mouse.

When you click your mouse the green outline and Detailed Alternative will be locked and a "Confirm" button and a "Cancel" button will appear. If you want to unlock the green outline just click on the "Cancel" button. If this is the alternative that you want most in this choice-situation, click on the "Confirm" button. Once you click "Confirm" you will not be able to change your selection.

Please wait to turn the page until you are instructed to do so.

Figure 14: Instructions Page 6 


\section{The Experiment: Your Choices}

In the experiment your job is to select the alternative that you want most from a list of nine alternatives. To make your job easier, the lists of alternatives are always arranged in a systematic way in the Selection Bar.

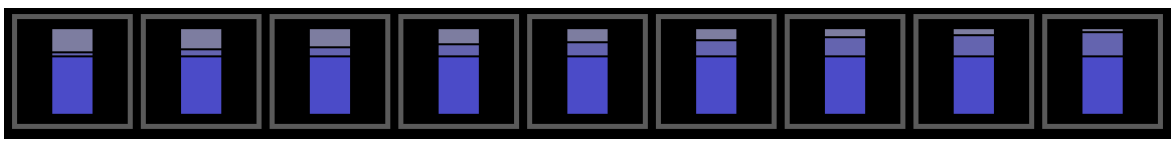

The Selection Bar depicted just above represents a choice-situation in which the probability of getting CHF 40 is the same for all of the nine alternatives. This is easy to see because the height of the dark blue boxes at the bottom of each alternative, the boxes that represent the probability you will get CHF 40, are the same height across the entire Selection Bar. However, as you move from left to right across this Selection Bar the probability you will get CHF 20 increases while the probability you will get CHF 0 decreases. Again, this is easy to see because of the way the alternatives are arranged in the Selection Bar: As you move from left to right the grey box at the top of the alternatives, the box representing the probability you will get CHF 0 , get smaller while the light-blue box in the middle of the alternatives, the box representing the probability you get CHF 20, gets bigger.

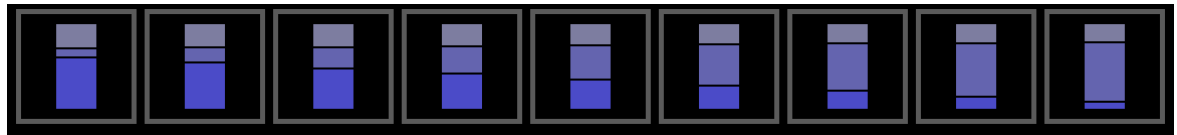

Most of the choice-situations in the experiment will be more complicated than the one just described. For example, in the Selection Bar depicted in the middle of this page, the right-most alternative has a large probability that you will get CHF 20 and small probabilities that you will get CHF 0 or 40 . As you move from right to left across this Selection Bar the probability you will get CHF 20 decreases while the probability you will get CHF 0 and the probability you will get CHF 40 both increase. The rate at which the three probabilities trade off against each other will vary from choice-situation to choice-situation. For the Selection Bar depicted in the middle of this page the tradeoff is tilted in favor of the probability of CHF 40 relative to the probability of CHF 0 . You can see this as you move from right to left across this Selection Bar because the probability of CHF 40 (dark blue boxes on the bottom) increases at a faster rate than the blocks representing the probability of CHF 0 (grey boxes on the top).

In contrast the Selection Bar depicted at the bottom of this page represents a choice-situation in which the tradeoff is tilted the other direction. The right-most alternative once again has a large probability of CHF 20. But, as you move from right to left the probability of CHF 0 increases at a faster rate than the probability of CHF 40.

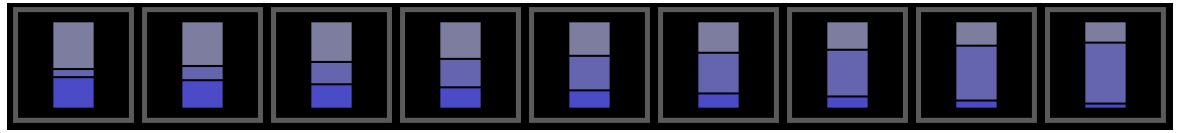

Please wait to turn the page until you are instructed to do so.

Figure 15: Instructions Page 7 


\section{Payments}

This section explains how your payments will be determined and how you will receive them. All payments will be in cash, today, here at the lab.

You will receive CHF 10 just for participating. You will also receive payment from one choicesituation that is randomly selected to count for actual payment. During the experiment you will select the alternative you want most from each of the choice-situations. Once you are finished making your selections the computer will randomly select one choice-situation to count for actual payment. The computer will display the choice-situation and the alternative you selected therein. You don't know which choice-situation will count for actual payment until after you have made all of your selections. And you won't be able to change your selection during the payment phase. So, your best strategy is to treat each choice-situation as if it counts for actual payment by looking at all of the alternatives available, and then selecting the alternative that you want most.

When you have finished making your selections, the computer will instruct you to press a key. The computer will then randomly select the one choice-situation that counts for actual payment and display the alternative you selected. When this screen appears please raise your hand to get an assistant's attention. He will come over and write down all of the details of your alternative on the back of your identification card. You will verify that he wrote the information correctly and then initial this card. He will then take your card to the cashier's desk. After you complete this part of the experiment there is a questionnaire for you to fill out. When you have finished with the questionnaire please get our attention so that we know you are ready to determine your payment from your alternative. However, please remain seated quietly until we call you to the cashier's desk. When you are called to the cashier's desk please take your questionnaire and your personal belongings.

Here is how the payments will work: Once you are at the cashier's desk you will roll two, 10-sided dice to determine which one of the three amounts you will get from your alternative. One of the 10-sided dice has 00 , $10,20, \ldots, 80,90$ on its faces. The other 10 -sided die has $0,1,2, \ldots, 8,9$ on its faces. After you roll the two dice we will add the two face-up numbers together and that total will be used to determine which amount you receive from your alternative. The only exception to this is how the number 100 is represented by the dice which is $00+0$.

To make clear how the roll of the two 10-sided dice will determine your payment we will use the alternative depicted to the right as an example. You can get CHF 0 , 20 , or 40 from this alternative. The probability of each of those amounts is $25 \%, 40 \%$, and $35 \%$, respectively. For this alternative, if you roll numbers 01 to 25 on the 10 sided dice you would receive CHF 0 . If you roll 26 to 65 you would receive CHF 20. If you roll 66 to 100 you would receive $\mathrm{CHF} 40$. The number-intervals and amounts will, of course, be adjusted for the specific alternative you selected in the one choice-situation that

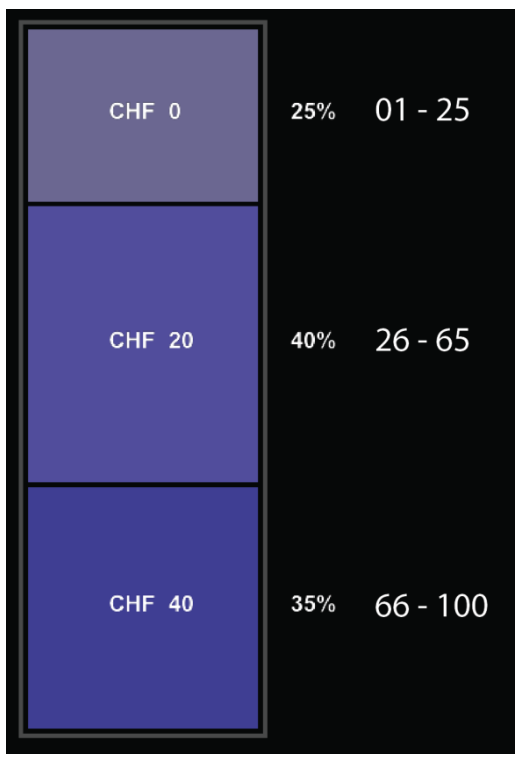
counts for actual payment. However, your roll of the 10-sided dice will determine which monetary amount you get from the alternative you selected in the choice-situation that counts for actual payment.

Figure 16: Instructions Page 8 